\title{
DOE A9024 Final Report
}

\section{Functional and Nanoscale Materials Systems: Frontier Programs of Science at the Frederick Seitz Materials Research Laboratory}

The scientific programs of the FSMRL supported under the DOE A9024 Grant consisted of four interdisciplinary research clusters, as described below. The clusters were led by Professors Tai Chiang (Physics), Jeffrey Moore (Chemistry), Paul Goldbart (Physics), and Steven Granick (Materials Science and Engineering). The completed work followed a dominant theme-Nanoscale Materials Systems-and emphasized studies of complex phenomena involving surfaces, interfaces, complex materials, dynamics, energetics, and structures and their transformations. A summary of our key accomplishments is provided below for each cluster.

\section{Research Accomplishments}

\section{Surface, Interface, and Thin Film Science}

Task 1: Atomistic, Quantum Electronic, and Nanoscale Phenomena Primary Participants: T. C. Chiang, G. Ehrlich, J. E. Greene, D. D. Johnson, I. G. Petrov, I. M. Robertson, I. K. Robinson, J. H. Weaver, and J. M. Zuo

\section{Task 2: Strongly Driven and Ultrafast Processes in Materials}

Primary Participants: R. S. Averback, D. G. Cahill, C. P. Flynn, D. D. Johnson, and J. M. Zuo

A number of important advances in the science of surfaces, interfaces, and thin film growth have taken place. The cluster research has led to the publication of 580 papers (including 66 in Physical Review Letters, 81 in Physical Review B, 9 in Science, 8 in Nature, and 43 in Applied Physics Letters). Among the students involved in the research, $48 \mathrm{Ph} . \mathrm{D}$. degrees were awarded. Selected highlights are presented below.

\section{Adatom Diffusion and Long Jumps}

The usual interpretation of surface diffusion is to view the motion of atoms on the surface as a random walk between nearest-neighbor sites. Recent efforts led by Ehrlich have been devoted to testing this idea, by looking for $\mathrm{W}$ atoms carrying out longer jumps on $\mathrm{W}(110)$. This is the first time that any long jump rates have been measured ${ }^{[1]}$. On 
W(110), four different kinds of jumps were identified, as indicated in Fig. 1: nearest-neighbor transitions $\alpha$, double jumps $\beta$ along $<111>$, and transitions along the horizontal axis, $\delta_{\mathrm{x}}$, and the vertical axis, $\delta_{\mathrm{y}}$. For the long jumps, the activation energies and prefactors were found to be much larger than for single nearest-neighbor transitions. A central question is - is this behavior typical or universal for metal surfaces? To test this, a detailed examination of the diffusion of Ir on W(110) was also performed, and the results showed similar behavior ${ }^{[2]}$. For both Ir and W, activation energies for the long jumps are quite high, so that they occur only at higher temperatures. However, vertical jumps for Ir take place at the same rate as for horizontal jumps, and this is quite different from the behavior of $\mathrm{W}$ atoms. A general conclusion for these systems is that long jumps, not nearest-neighbor transitions, are responsible for almost all diffusion events above $400 \mathrm{~K}$. Thus, the traditional

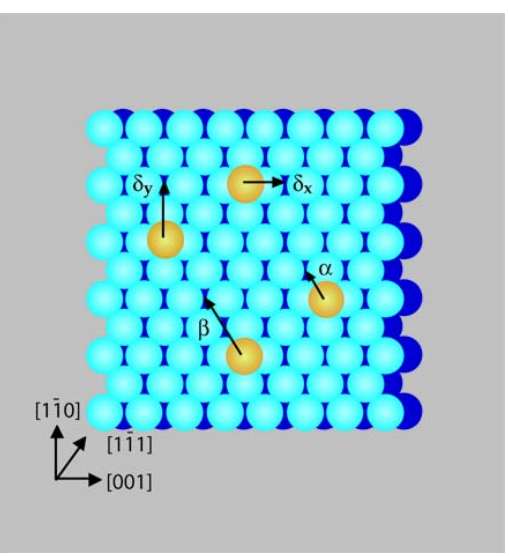

Fig. 1. Processes contributing to diffusion on $\mathrm{W}(110)$ : $\alpha$ jumps between nearest-neighbor sites, $\beta$ jumps to a site along $<111>$ direction two spacings from the origin, and horizontal $\delta_{\mathrm{x}}$ and vertical $\delta_{\mathrm{y}}$ jumps. view of diffusion must be refined.

\section{Step and Island Structure and Dynamics}

Atomic diffusion is intimately related to step motion, which is a subject of study by Flynn, Chiang, Greene, and Petrov. An example is the work of Flynn using step fluctuation spectroscopy in a LEEM to determine surface mass diffusion coefficients $D_{s}$ for the model surfaces $\operatorname{Pt}(111)^{[3]}, \mathrm{Au}(111)^{[4]}, \operatorname{Pd}(111)^{[5]}, \mathrm{Ni}(111)$ and $\mathrm{Mo}(011)^{[6]}$. Three other surfaces, $\mathrm{Cu}(111), \mathrm{Ag}(111)$ and $\mathrm{Fe}(011)$, are currently under study. Near $T_{m} / 2$ (one half of the melting temperature), the relaxation times vary as $q^{3}$, with $q$ the fluctuation wave vector ${ }^{[7,8]}$. This identifies the kinetics unambiguously with surface diffusion over the terraces. These results increase by a substantial factor the total available data for surface mass diffusion on terraces. The processes are believed to involve adatom hopping. The measured $D_{s}$ for $\mathrm{Au}(111), \operatorname{Pt}(111)$, and $\mathrm{Pd}(111)$ as a function of $T_{m} / T$ are similar, and differ greatly from the corresponding bulk mass diffusion coefficients $D_{b}$, which, however, exhibit their own homologous dependence on $T_{m} / T$. The homologous behavior for the bulk has long been recognized, but remains poorly explained. The fact that close packed surfaces exhibit similar mass diffusion coefficients, when viewed as homologous functions of $T_{m} / T$, is revealed for the first time in this research ${ }^{[9]}$. The importance of this result, if borne out by further research, is that surface mass diffusion, and the constraints it imposes on important processes such as molecular beam growth, ion erosion, etc. can, for the first time, be estimated for all close packed metal surfaces. Furthermore, the homologous character of bulk and surface diffusion gives rise to important universal behavior ${ }^{[10]}$ that appears to link a wide variety of metals. In processes like epitaxial growth, surface smoothing, step fluctuations, and erosion that depend on both bulk and surface diffusion, the behavior becomes universal. 
To explore erosion and growth on these same surfaces, a temperature range is selected in which diffusion is sufficiently fast that surface step structure is visibly (by LEEM) retained after erosion by a $1 \mathrm{keV} \mathrm{Ar}^{+}$ion beam. The erosion mainly causes uphill step flow, but with an evolution of step profile that is richly detailed. Three images for $\operatorname{Pd}(111)$ in Fig. 2 show (a) how, in initial erosion, the flow past erosion-induced features causes chains of correlated structure on successive steps, which in (b) and (c) merge to form sinusoidal ripples ${ }^{[11]}$. As in spinodal processes, the wavelength is determined by diffusive decay. Upon further erosion the profiles become asymmetric with downhill points resembling Mullins-Sekerka interfaces, which correspond to diffusion profiles limited
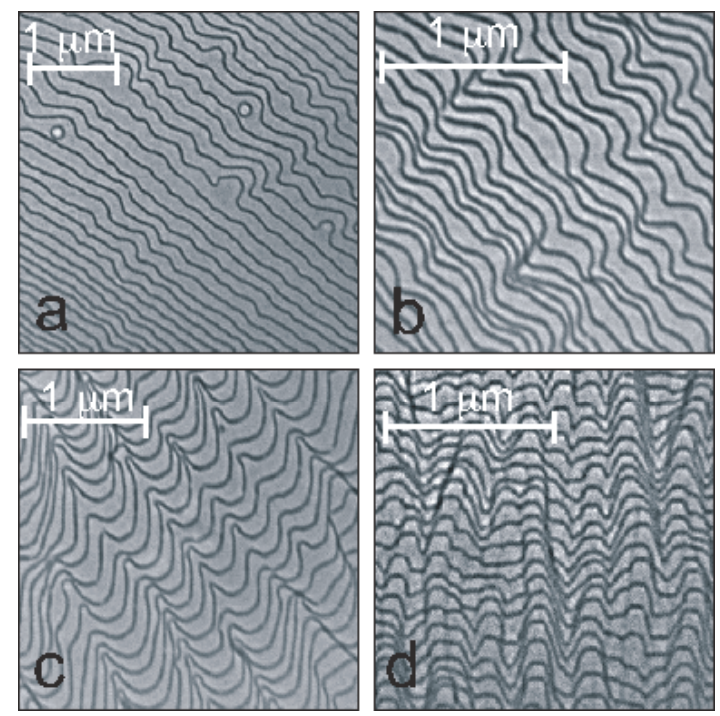

Fig. 2. (a)-(c) Surface step profiles on Pd (111) with increasing erosion. (d) $\mathrm{Ni}(111)$. by surface defects, probably advacancies, constrained by an Ehrlich-Schwoebel barrier at the steps. The universal character is indicated in (d) by a comparison with $\mathrm{Ni}(111)$; these detailed features recur on $\mathrm{Ni}(111)$, $\operatorname{Pd}(111)$, and $\operatorname{Pt}(111)$ at closely similar values of $T / T_{m}$, namely, $0.43,0.48$ and 0.40 .

Substantial work has also been performed on surfaces of transition metal nitrides. Greene, Petrov, and others are developing a robust quantitative predictive model for microstructural and surface morphological evolution ${ }^{[12,13,14,15]}$. The model requires, as input, adatom transport activation energies as well as island line tensions, step energies, step stiffnesses, kink energies, edge atom mobilities, and interlayer mass transport as a function of local island orientation. For the first time, all of these quantities are extracted from numerical analysis of high-temperature STM and LEEM data for the (001) and (111) surfaces of $\operatorname{TiN}^{[16,17,18,19,20,21]}$. The parameters are now being used as input in a level-set model designed to simulate step and island dynamics. Numerical results are in excellent agreement with available in-situ STM time-sequence images. The model also allows simulations on time scales and under experimental conditions not easily accessible to experiments. 


\section{Adsorbate Interactions}

An important issue for surface and thin film processing is surface stability, which can be modified by adsorption. This effect has been studied by Weaver in detail for the adsorption of $\mathrm{Cl}, \mathrm{Br}$, and I on $\mathrm{Si}(100)-(2 \times 1)^{[22,23,24,25,26]}$. As can be seen in Fig. 3, the surface becomes destabilized through adsorbate-adsorbate steric repulsive interactions. These interactions increase with increasing halogen size, making it thermodynamically favorable for the surface to roughen rather than to remain planar. For a deeper understanding of these observations, Johnson has performed electronic structure calculations $^{[27]}$. The results indicate a crossover in surface patterning depending on halogen used, as observed from experiment by Weaver $^{[27]}$. In addition, a scaling property of

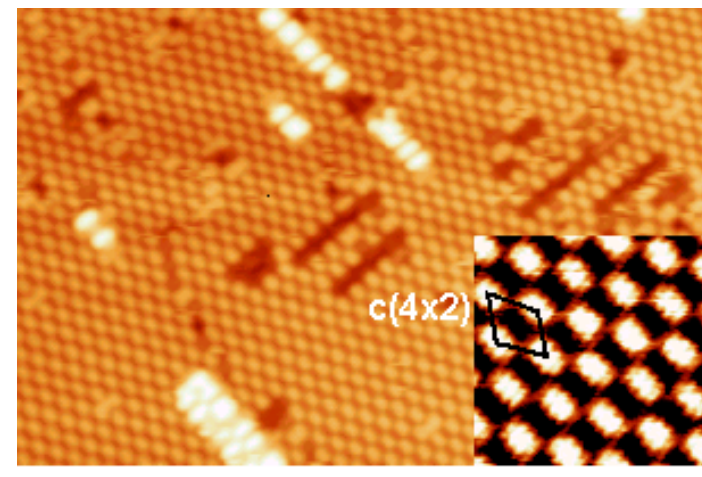

Fig. 3. STM image of $\mathrm{Si}(100)$ with $\sim 0.5 \mathrm{ML}$ of I. The inset shows a $\mathrm{c}(4 \times 2)$ pattern with iodine bonded to alternating dimers to minimize I-I repulsive interactions (bare dimers are dark). This surface roughens, producing terrace vacancies, terrace vacancy lines, and islands on the terrace. the steric-repulsion energies is found. A simple model has been developed that explains the scaling and crossover and shows that the apparent steric-repulsion really is a consequence of surface bonding effects. Halogen termination of $\mathrm{Si}$ and Ge surfaces is also useful for a different purpose. Chiang and his collaborators have examined, using STM, the atomic structure and chemical composition of Ge films grown on Si by atomic layer epitaxy using digermane as the source gas ${ }^{[28]}$. Termination of the as-grown surfaces by $\mathrm{Cl}$ enhances the contrast between $\mathrm{Si}$ and $\mathrm{Ge}$ atoms, thus permitting a detailed statistical analysis of the $\mathrm{Si}$ and Ge surface atom distributions at various stages of the growth. The interfacial chemical profile is shown to be graded, but interlayer exchange occurs only at the growing surface. In related work, Greene and coworkers have developed a low energy ion scattering technique for the first direct measurements of strain depth profiles in $\mathrm{Ge} / \mathrm{Si}(001)$ nanostructures ${ }^{[29]}$.

\section{Clusters, Epitaxy, and Grain Boundaries}

Adatom motion and interaction can result in cluster formation. While STM allows a detailed view of surface features, it does not provide information about the internal lattice structure of clusters. Zuo and Robertson use TEM in combination with molecular dynamics simulations to examine structural issues related to nanocluster formation and evolution $^{[30,31,32,33]}$. In a study of Ag deposited on H-terminated Si(111), Zuo discovered novel and complex interfacial behavior at the nanometer scale. As the interfacial area approaches the size of a Coincidence Site Lattice (CSL) cell, $\sim 1.6 \mathrm{~nm}$, the structure evolves due to competition between strain and the tendency toward commensuration. The details of the structural evolution depend on temperature. Deposition at room temperature favors the formation of multi-twinned cluster structures, while at $\sim 200{ }^{\circ} \mathrm{C}$ the clusters are mostly epitaxial. Annealing to $\sim 400{ }^{\circ} \mathrm{C}$ causes the clusters to evolve into almost perfect epitaxial crystalline $\mathrm{Ag}$ with a coincidence lattice and an orientation of $\mathrm{Ag}(100)|| \operatorname{Si}(100)$ 
and $\operatorname{Ag}[011]|| \operatorname{Si}[011]$, as illustrated in Fig. $4^{[34]}$. These results suggest that epitaxy is not preferred at the early stage of cluster nucleation, and interfacial effects subsequently lead to cluster reorientation and crystallization. These studies significantly improve our understanding of interfacial evolution associated with epitaxial growth for mismatched metal-semiconductor systems.

\section{Advances in Diffraction and Scattering Methods}

Coherent diffraction methods are under active development within this cluster, mainly by Zuo and Robinson, using electron beams and $\mathrm{x}$-rays, respectively. These methods are based on direct inversion of diffraction data to yield real-space images ${ }^{[35]}$. The work of Zuo has focused on large molecules and surface clusters, and a recent study of carbon nanotubes has demonstrated atomic resolution $^{[36,37]}$. The work of Robinson is performed at APS, using the coherent diffraction beamline on Sector 34. Innovative use of x-ray optics leads to micron-sized beams that preserve coherence. With a coherent beam, each atom in the system can be treated as a phase object, and atomic displacements as small as a fraction of an Ångstrom can be detected. Work so far has focused on metal nanocrystals ${ }^{[38,39,40]}$ and $\mathrm{Ge}_{x} \mathrm{Si}_{1-\mathrm{x}}$ films grown on $\mathrm{Si}^{[41,42]}$. As an example, Fig. 5 presents a coherent diffraction pattern from a $\mathrm{Ag}$ nanocrystal. The work on $\mathrm{Ge}_{\mathrm{X}} \mathrm{Si}_{1-\mathrm{x}}$ films uses samples grown by Spila and Greene using gasphase CVD. The films, with thicknesses of a few hundred nanometers, contain a quadrangular array of misfit dislocations at the interface, each of

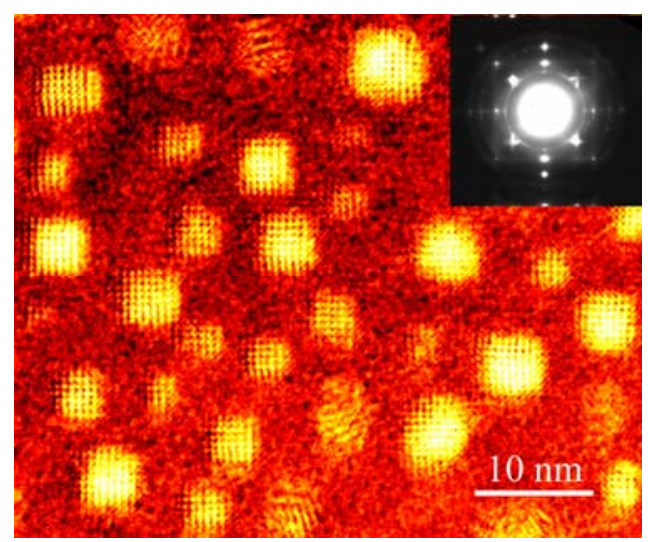

Fig. 4. Epitaxial Ag nanocrystals grown on hydrogen-terminated $\mathrm{Si}(100)$. The nanocrystals were formed by room temperature deposition followed by annealing at $\sim 450{ }^{\circ} \mathrm{C}$. The inset is an electron diffraction pattern. The crosslike spots are $\mathrm{Ag}\{200\}$ reflections.

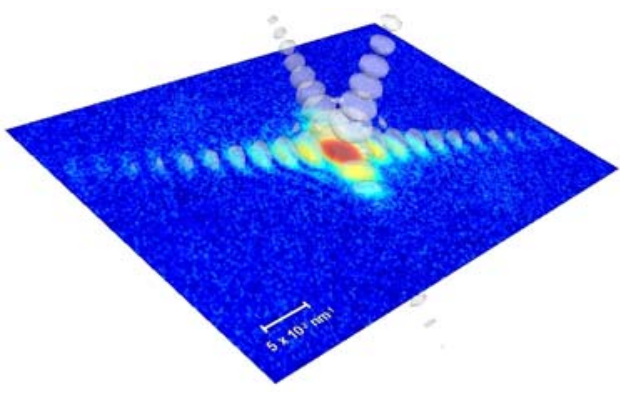

Fig. 5. Coherent x-ray diffraction pattern of a $160 \mathrm{~nm} \mathrm{Ag}$ nanocube. The slice through reciprocal space captured by the experiment is illustrated in the intersection of the data image with a full three-dimensional simulation of the diffraction amplitudes from the nanocube. The simulation is represented by the translucent isosurface of amplitude in the picture. which glides to the surface along $\{111\}$ planes.

The measured coherent diffraction patterns reveal rich structures that can be indexed and related to the defect strain field. Detailed numerical analysis is underway, and a new algorithm is under development in collaboration with Zuo. In related work, Chiang and Zuo are developing methods based on x-ray and electron-beam thermal diffuse scattering to extract information about lattice dynamics and phase transitions in materials ${ }^{[43,44,45,46]}$. 


\section{Quantum Confinement and Electron-Lattice Coupling}

Geometric confinement of electrons, symmetry reduction, and boundary effects in thin films can lead to interesting and useful properties. These effects are being investigated by Chiang using angle-resolved photoemission and x-ray diffraction, with theoretical support from Johnson, Martin, and others. Recent work has led to the surprising discovery that atomically uniform films of $\mathrm{Pb}$ can be grown on $\mathrm{Si}(111)$ despite

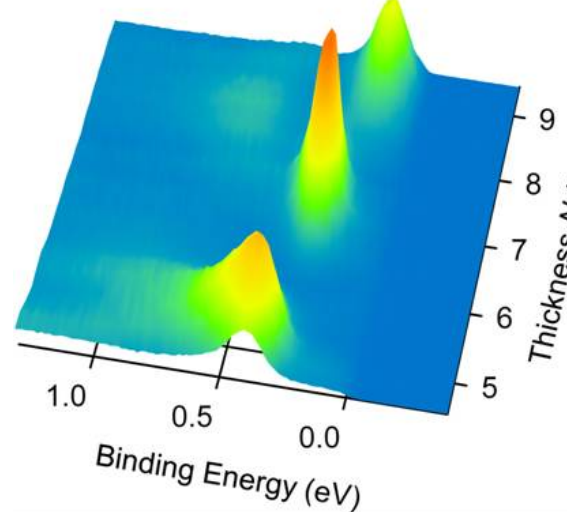

Fig. 6. Normal emission photoelectron intensity as a function of film thickness and binding energy for $\mathrm{Pb}$ films grown on $\mathrm{Pb}$-terminated Si(111). The discrete quantum-well peak evolution shows that films are atomically uniform at integer monolayer thicknesses. a large lattice mismatch ${ }^{[47]}$. Figure 6 presents angleresolved photoemission spectra taken along the surface normal direction for $\mathrm{Pb}$ films grown on $\mathrm{ab}$ terminated $\mathrm{Si}(111)-\sqrt{3} \times \sqrt{3}$ surface. The discrete peak evolution indicates atomic layer uniformity at integer monolayer thicknesses. Sharp quantum well peaks are seen for film thickness $N=5,7$, and 9 monolayers (ML), but not for $N=6$ and 8. A calculation of the electronic structure yields a period of quantum oscillations of $2.2 \mathrm{ML}$, implying a nearly bilayer modulation of properties. Indeed, films with $N=6$ and 8 are much more thermally stable than those with $N=5,7$, and 9. Electron confinement can also lead to strain. Friedel oscillations in charge density, with the same $2.2 \mathrm{ML}$ period, result in an oscillatory electronic force on the atomic cores and a corresponding strain field. This effect has been confirmed by x-ray diffraction using the UNICAT facility on Sector 33 at the Advanced Photon Source ${ }^{[48]}$. So far, data analysis has relied on

least-squares fittings. Chiang and Robinson are planning coherent diffraction measurements for direct determination of the strain field.

The energetics for $\mathrm{Pb}$ film deposition on $\mathrm{Si}(111)-(7 \times 7)$ is significantly different from that for deposition on the $\sqrt{3} \times \sqrt{3} \mathrm{~Pb}$-terminated surface, suggesting the importance of boundary effects. The initial growth of $\mathrm{Pb}$ on the $(7 \mathrm{x} 7)$ substrate at $180 \mathrm{~K}$ is a flat wetting monolayer, followed by the formation of islands of a fairly uniform height of $5 \pm 1$ ML (for a total island height of $6 \mathrm{ML}$ counting from the substrate surface). These magic-height islands expand to cover the surface, and layer-by-layer growth takes over on further deposition. This highly unusual growth behavior is explained by a first-principles calculation. Figure 7 shows the calculated surface energy per atom for freestanding $\mathrm{Pb}$ films and for $\mathrm{Pb} / \mathrm{Si}(111)$. The results exhibit Friedel-like damped oscillations and a beating pattern, caused by the slight difference of the $2.2 \mathrm{ML}$ oscillation period from 2 ML. The main difference between the freestanding and

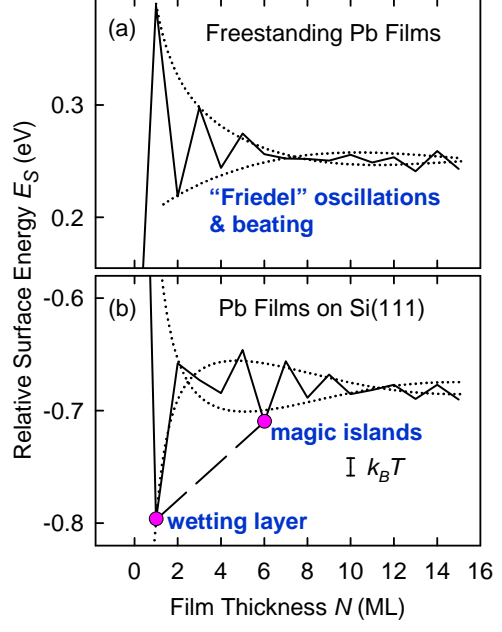

Fig. 7. Calculated surface energy for (a) freestanding and (b) supported $\mathrm{Pb}$ films. 
supported films is the phase of the beating function. For the supported films, the absolute minimum of the surface energy at $N=1$ favors a wetting layer. For coverages 1-6 ML, the system phase separates into a combination of $N=1$ and 6 , as indicated by the dashed line in Fig. 7(b). Thus, the observed magic island formation at $N=6$ can be attributed to this quantum phase separation effect ${ }^{[49]}$. These electronic effects can likewise play an importance role in the physics of clusters; the implication is being investigated by Robinson and Zuo.

Other members of the group are also interested in quantum confinement and ways to synthesize nanostructures. For example, Weaver has developed a unique soft-landing cluster deposition technique that can be used to synthesize a wide variety of nanostructures $^{[50]}$. It is based on physical vapor deposition onto thin solid rare gas buffer layers that have been condensed on a substrate of choice. That deposition leads to the spontaneous formation of clusters. Subsequent warm-up results in buffer layer desorption and, ultimately, the delivery of the clusters to the surface. This technique makes it possible to create unique nanoparticle-substrate combinations where fundamental issues related to stability, wetting, and interface reactions are explored. Significantly, the size of the delivered particles can be controlled through the thickness of the buffer layer since the particles diffuse and coalescence on the buffer layer during its desorption. Experimental studies using the TEM of the CMM and modeling have shown that this random-walk diffusion of structures derived from 10's to 10's of thousands of atoms is related to the elementary excitations of the buffer layer, namely the phonons $^{[51,52]}$.

\section{Driven Systems}

Systems that are strongly driven by particle or energy deposition can display novel features and properties. To explore the possibilities, Cahill and Greene employ laser processing to modify surfaces and films ${ }^{[53,54,55,56]}$. A recent experiment on the controlled pulsed illumination of thin thermally-grown oxide layers on $\mathrm{Si}$ has shed light on the process of delamination by thermal stress due to differential expansion $^{[57]}$. The image in Fig. 8 shows the cross section of a laser blister, which is unexpected and suggests that the work of adhesion of the $\mathrm{SiO}_{2} /$ liquid-Si interface is strongly suppressed at the extremely high temperatures, $\sim 3000 \mathrm{~K}$, that are produced by laser processing. The results illustrate a novel means of modifying surface morphology. Laser texturing has also been employed to create $\mathrm{Ge}(001)$ substrates with a wide range of surface orientations for fundamental studies of pattern formation during low temperature crystal growth ${ }^{[58]}$. Vicinal surfaces oriented in the $<110>$ direction form relatively high aspect-ratio ripple morphologies. Surprisingly, $<100>$ miscuts completely suppress the instability. The results demonstrate that vicinal surfaces can facilitate the formation of abrupt and smooth

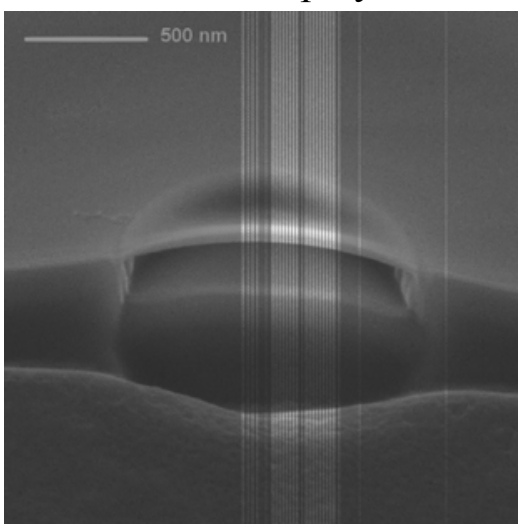

Fig. 8. SEM image of the cross section, prepared by focused-ionbeam machining, of a laser blister. The $25 \mathrm{~nm}$ thick Si oxide layer has separated from the $\mathrm{Si}$ substrate, leaving behind a void 2 $\mu \mathrm{m}$ in diameter and $200 \mathrm{~nm}$ deep. 
interfaces at low temperatures. Extensions of the existing $1+1$ dimensional theory to $2+1$ dimensions cannot explain the anisotropy.

Ion beam irradiation is employed for film processing by Averback and Cahill. Planar metal films deposited on dielectric substrates are typically thermodynamically unstable and when heated roughen by a process of dewetting. In most studies of dewetting transitions, films are heated homogeneously and dewetting occurs simultaneously over large areas of the specimen. Thermal spikes produced by individual heavy-ion impacts, on the other hand, are highly localized both in space (nanometers) and time (picoseconds). Recent work of this group has demonstrated the robustness of ion-beam induced dewetting for creating controlled, nanostructured morphologies ${ }^{[59]}$. The process is remarkably insensitive to temperature, ion species, and ion energy as long as the ion energy is sufficiently high for the ion to penetrate through the metal film.

In related work, Averback has developed a firm understanding of beam-induced modifications of surface morphology and stress in irradiated amorphous, nanocrystalline and polycrystalline films ${ }^{[60,61]}$. For amorphous films, combined AFM and molecular dynamics studies revealed that the morphology of the irradiated film can be understood on the basis of a dynamic competition between ballistic roughening and viscous-flowinduced smoothening. The ballistic roughening derives from two sources of noise: sputtering and crater formation. The former gives rise to atomic scale roughness while the latter causes roughening and surface patterning with a length scale of 3-10 nm. A detailed analysis of the spectral power density as a function of wave vector reveals the effects of these noise terms and relaxation by viscous flow ${ }^{[62]}$.

\section{Complex Nanostructures and Microstructures}

Petrov, Greene, Cahill, and Chiang are interested in films of transition metal nitrides which are important for diffusion barrier and wear- and corrosion-resistant coating applications. The layers are prepared by a novel magnetically-unbalanced UHV magnetron reactive sputtering deposition method or by laser ablation. The sputtering method allows ion-to-neutral flux ratios to be varied over wide ranges at low ion energies. XRD, TEM, XTEM, and in-situ high-temperature STM and LEEM studies have been carried out to characterize the growth and microstructure evolution at the atomic scale $^{[15,21,63,64,65,66,67,68,69,70,71,72,73]}$. Figure 9 shows that independent tuning of the incident ion energy and flux makes it possible to control structure, and hence physical properties, at the nanoscale.

Petrov and Greene have developed a novel, widely applicable approach for systematically and rapidly obtaining very-high-index substrate orientations which allow the directed growth of highly-ordered self-organized nanostructures $^{[74]}$. A structural model is employed to evaluate the tilt and azimuthal angles required to isolate



Fig. 9. XTEM image of a $\mathrm{Ti}_{0.8} \mathrm{Ce}_{0.2} \mathrm{~N}$ film grown with incident ion energy of (a) 14 and (b) $45 \mathrm{eV}$. Dashed lines outline TiN-rich (ordered) and CeN-rich (disordered) regions. 
families of template structures with nanoscale facetting arrays (for example, hill-andvalley structures), on which desired shape-, size-, and position-controlled nanostructures can be grown. The results are used as input for computer-controlled focused ion-beam (FIB) fabrication of substrates. Figure 11 presents recent results on the synthesis of Ge nanowires and teardrops, with uniform shape, dimension, and periodicity, on $\operatorname{Si}(173100$ $373)$ and related surfaces. The $\left(\begin{array}{lll}173 & 100 & 373\end{array}\right)$ plane is a member of the family $(k 100$ $200+k)$ composed of a (113)/(517) hill-and-valley structure, in which the ratio of the two facet widths can be controllably varied.

\section{Selected Publications:}

[1] G. Antczak and G. Ehrlich, "Long Jump Rates in Surface Diffusion: W on W(110)," Phys. Rev. Lett. 92, 166105 (2004).

[2] G. Antczak and G. Ehrlich, "Long Jumps in Diffusion of Iridium on W(110))," Phys Rev. B (in press).

[3] M. Ondrejcek, W. Swiech, G. Yang, and C.P. Flynn, "Crossover from bulk to surface diffusion in the fluctuations of step edges on Pt (111)," Phil. Mag. Lett. 84, 69417 (2004).

[4] M Ondrejcek, M Rajappan, W Swiech, and C.P. Flynn, "Step Fluctuation Spectroscopy of Au (111) by LEEM," Surf. Sci. 574, 111 (2005).

[5] M. Ondrejcek, W. Swiech and C.P. Flynn, "Studies of step stiffnesses and relaxation on $\operatorname{Pt}(111), \operatorname{Pd}(111)$ and Mo(011)," Surf. Sci. 566-568, 160 (2004).

[6] M. Ondrejcek, W. Swiech, C.S. Durfee and C.P. Flynn, "Step fluctuations and step interactions on Mo (011)," Surf. Sci. 541, 31 (2003).

[7] C.P. Flynn, "Step-edge fluctuations on crystal surfaces," Phys. Rev. B 66, 155405 (2002).

[8] H.C. Jeong, E.D. Williams, "Steps on surfaces: experiment and theory," Surf. Sci. Rep. 34, 171 (1999).

[9] M. Ondrejcek, M. Rajappan, W. Swiech and C.P. Flynn, "LEEM investigations of kinetics and energetics on clean close packed metal surfaces," J. Phys. Conden. Matter (in press).

[10] C.P. Flynn, "Point defect reactions at surfaces and in bulk metals," Phys. Rev. B (in press).

[11] M. Ondrejcek, W. Swiech, M. Rajappan and C.P. Flynn, "Ripples formed in the sputter erosion of Pd (111)," J. Phys. Conden. Matter 15, L735 (2003).

[12] F.H. Beauman, D.L. Chopp, G.H. Gilmer, J.E. Greene, H. Huang, S. Kodambaka, I. Petrov, P. O'Sullivan, and T. Diaz de la Rubia, "Multi-scale Modeling of Thin Film Deposition: Applications to Si Device Processing," MRS Bull. 26,182 (2001).

[13] D. Gall, S. Kodambaka, M.A. Wall, I. Petrov, and J. E. Greene, "Pathways of Atomistic Processes on TiN(001) and (111) Surfaces during Film Growth: an Ab-Initio Study," J. Appl. Phys. 93, 9086 (2003).

[14] I. Petrov, P.B. Barna, L. Hultman, and J.E. Greene, "Microstructural Evolution during Film Growth," J. Vac. Sci. Technol. 21, S117 (2003).

[15] M.A. Wall, D.G. Cahill, I. Petrov, D. Gall, and J.E. Greene, "Nucleation Kinetics during Homoepitaxial Growth of TiN(001) by Reactive Magnetron Sputtering," Phys. Rev. B 70, 035413 (2004). 
[16] S. Kodambaka, V. Petrova, A. Vailionis, P. Desjardins, D.G. Cahill, I. Petrov, and J.E. Greene, "TiN(001) and TiN(111) Island Coarsening Kinetics: In-situ Scanning Tunneling Microscopy Studies," Thin Solid Films 392, 164 (2001).

[17] S. Kodambaka, V. Petrova, S.V. Khare, D.D. Johnson, I. Petrov, and J.E. Greene, "Absolute TiN(111) Step Energies from Analysis of Anisotropic Island Shape Fluctuations," Phys. Rev. Lett. 88, 146101 (2002).

[18] S.V. Khare, S. Kodambaka, D.D. Johnson, I. Petrov, and J.E. Greene, "Determining Absolute Orientation-Dependent Step Energies: A General Theory for the Wulff-

Construction and for Anisotropic Two Dimensional Island Shape Fluctuations," Surf. Sci. 522, 75 (2003).

[19] S. Kodambaka, V. Petrova, S.V. Kharẹ, D. Gall, A. Rockett, I. Petrov, and J.E. Greene, "Size-Dependent Detachment-Limited Decay Kinetics of Two-Dimensional TiN Islands on TiN(111)," Phys. Rev. Lett. 89, 176102 (2002).

[20] S. Kodambaka, S.V. Khare, V. Petrova, D.D. Johnson, I. Petrov, and J.E. Greene, "Absolute Orientation-Dependent Anistropic TiN(111) Island Step Energies and Stiffnesses from Shape Fluctuation Analyses," Phys. Rev. B 67, 035409 (2003). [21] S. Kodambaka, S.V. Khare, W. Święch, K. Ohmori, I. Petrov, J.E. Greene, "Dislocation-Driven Surface Dynamics on Solids," Nature 429, 49 (2004).

[22] G.J. Xu, E. Graugnard, V. Petrova, K.S. Nakayama, and J.H. Weaver, "Dynamics of Surface Roughening of Cl-terminated Si(100)-2x1 at 700 K," Phys. Rev. B 67, 125320 (2003).

[23] G.J. Xu, K.S. Nakayama, B.R. Trenhaile, C.M. Aldao, and J.H. Weaver, "Equilibrium Morphologies for Cl-Roughened Si(100) at 700-750 K: Dependence on Cl Concentration," Phys. Rev. B 67, 125321 (2003).

[24] G.J. Xu, E. Graugnard, B.R. Trenhaile, K.S. Nakayama, and J.H. Weaver, "Atom Vacancy Lines and Surface Patterning: The Role of Stress for Br- Si(100)-(2x1) at 700 K," Phys. Rev. B 68, 75301(2003).

[25] G.J. Xu, S.V. Khare, K.S. Nakayama, C.M. Aldao, and J.H. Weaver, "Step Free Energies, Surface Stress, and Adsorbate Interactions for Cl-Si(100) at 700 K," Phys. Rev. B 68, 235318 (2003).

[26] G.J. Xu, A.W. Signor, A. Agrawal, K.S. Nakayama, B.R. Trenhaile, and J.H. Weaver, "Halogen Adatom and Pair Diffusion on Si(100) and the Role of Defects," Surf. Sci. (in press).

[27] G.J. Xu, N.A. Zarkevich, A. Agrawal, A.W. Signor, B.R. Trenhaile, D.D. Johnson, and J.H. Weaver, "Cross-over Energetics for Halogenated Si(100): Vacancy Line Defects, Dimer Vacancy Lines, and Atom Vacancy Lines," Phys. Rev. B (in press). [28] D.-S. Lin, J. L. Wu, S.-Y. Pan, and T.-C. Chiang, "Atomistics of Ge deposition on Si(100) by atomic layer epitaxy," Phys. Rev. Lett. 90, 046102 (2003).

[29] D.W. Moon, H.I. Lee, B. Cho, Y.-L Foo, T. Spila, S. Hong, C.P. Liu, M.A. Wall, and J.E. Greene, "Direct Measurements of Strain Depth Profiles in Ge/Si(001) Nanostructures," Appl. Phys. Lett. 83, 5298 (2003).

[30] J. M. Zuo and B.Q. Li, "Nanostructure Evolution During Cluster Growth: Ag on HTerminated Si(111) Surfaces," Phys. Rev. Lett. 88, 255502 (2002).

[31] B. Q. Li and J.M. Zuo, "The development of epitaxy of nanoclusters on latticemismatched substrates: Ag on H-Si(111) surfaces," Surf. Sci. 520, 7 (2002). 
[32] J. K. Bording, Y.F. Shi, B.Q. Li and J.M. Zuo, "Size- and shape-dependent energetics of nano-crystal interfaces: experiment and simulation," Phys. Rev. Lett. 90, 226104 (2003).

[33] Chun-Ming Li, I. M. Robertson, M. L. Jenkins, J. L. Hutchison, and R. C. Doole, "In situ TEM observation of the nucleation and growth of silver oxide nanoparticles," Micron, 36, 9 (2005).

[34] B. Q. Li and J.M. Zuo, "Shape and structure transition of Ag nanoparticles on Si(100)," Phys. Rev. B, (submitted).

[35] See, for example, J. R. Fienup, "Phase retrieval algorithms: a comparison," Applied Optics 21, 2758 (1982).

[36] J.M. Zuo, I. Vartanyants, M. Gao, R. Zhang and L.A. Nagahara, "Atomic Resolution Imaging of A Single Double-Wall Carbon Nanotube From Diffraction Intensities," Science, 300, 1419 (2003).

[37] J.M. Zuo, M. Gao, J. Tao, B.Q. Li, R. Twesten and I. Petrov, "Coherent Nano-Area Electron Diffraction," Microscopy Research Techniques (Invited), 64, 347 (2004). [38] I. K. Robinson, I. A. Vartanyants, G. J. Williams, M. A. Pfeifer and J. A. Pitney, "Reconstruction of the Shapes of Gold Nanocrystals using Coherent X-ray Diffraction," Phys. Rev. Lett. 87, 195505 (2001).

[39] G. J. Williams, M. A. Pfeifer, I. A. Vartanyants and I. K. Robinson, "Threedimensional Imaging of Microstructure in Gold Nanocrystals," Phys. Rev. Lett. 90, 175501 (2003).

[40] Ian K. Robinson, Franz Pfeiffer, Ivan A. Vartanyants, Yugang Sun, and Younan Xia, "Enhancement of Coherent X-ray Diffraction from Nanocrystals by Introduction of X-ray Optics," Opt. Express 11, 2329 (2003).

[41] F. Pfeiffer, W. Zhang and I. K. Robinson, "Coherent Grazing Exit X-ray Scattering Geometry for Probing the Structure of Thin Films," App. Phys. Lett. 84, 1847 (2004). [42] I. K. Robinson, Y. Da, T. Spila, and J. E. Greene, "Coherent Diffraction Patterns of Individual Dislocation Strain Fields," J. Appl. Phys. D (submitted).

[43] M. Holt, Z. Wu, Hawoong Hong, P. Zschack, P. Jemian, J. Tischler, Haydn Chen, and T.-C. Chiang, "Determination of phonon dispersions from X-ray transmission scattering: the example of silicon," Phys. Rev. Lett. 83, 3317 (1999).

[44] M. Holt, P. Zschack, H. Hong, M. Y. Chou, and T.-C. Chiang, "X-ray studies of phonon softening in TiSe2," Phys. Rev. Lett. 86, 3799 (2001).

[45] J.M. Zuo and J. Tao, "Nanometer-sized Regions of Charge Ordering and Charge Melting in $\mathrm{La}_{2} / 3 \mathrm{Ca}_{1} / 3 \mathrm{MnO}_{3}$ Revealed by Electron Micro-diffraction," Phys. Rev. B Rapid Comm. 63, 060407 (2001).

[46] J. Tao and J.M. Zuo, "Nanoscale Phase Competition during Charge Ordering in Intrinsically Strained La0.33Ca0.67MnO3," Phys. Rev. B, Rapid Comm. B 69, 180404 (2004).

[47] M. Upton, C. M. Wei, M. Y. Chou, T. Miller, and T.-C. Chiang, "Thermal stability and electronic structure of atomically uniform films of $\mathrm{Pb}$ on $\mathrm{Si}(111)$," Phys. Rev. Lett. 93, 026802 (2004).

[48] P. Czoschke, H. Hong, L. Basile, and T.-C. Chiang, "Quantum oscillations in the layer structure of thin metal films," Phys. Rev. Lett. 91, 226801 (2003). 
[49] H. Hong, C.-M. Wei, M. Y. Chou, Z. Wu, L. Basile, H. Chen, M. Holt, and T.-C. Chiang, "Alternating layer and island growth of $\mathrm{Pb}$ on $\mathrm{Si}$ by spontaneous quantum phase separation," Phys. Rev. Lett. 90, 076104 (2003).

[50] J.H. Weaver and G.D. Waddill, "Cluster-Assembly of Interfaces: Nanoscale Engineering," Science 251, 1444 (1991).

[51] V.N. Antonov, J.S. Palmer, A.S. Bhatti, and J.H. Weaver, "Nanostructure Diffusion and Aggregation on Desorbing Rare-gas Solids: Slip on an Incommensurate Lattice," Phys. Rev. B 68, 205148 (2003).

[52] V.N. Antonov, J.S. Palmer, P.S. Waggoner, A.S. Bhatti, and J.H. Weaver, "Nanoparticle Diffusion on Desorbing Solids: The Role of Elementary Excitations in Buffer-layer-assisted Growth," Phys. Rev. B 70, 045406 (2004).

[53] T. Schwarz-Selinger, Y.-L. Foo, D.G. Cahill, and J.E. Greene, "Surface Mass

Transport and Island Nucleation during Growth of Ge on Laser Textured Si(001)," Phys. Rev. B 65, 125317 (2002).

[54] B. Cho, T. Schwarz-Selinger, K. Ohmori, D.G. Cahill, and J.E. Greene, "The Effect of Growth Rate on the Spatial Distributions of Dome Shaped Ge Islands on Si(001)," Phys. Rev. B 66, 195407 (2002).

[55] F. Watanabe, D.G. Cahill, Sukwon Hong, and J.E. Greene, "Strained Layer Instabilities on Vicinal Surfaces: Ge0.8Sio.2 Epitaxy on Laser Textured Si(001)," Appl. Phys. Lett. 85, 1238 (2004).

[56] F. Watanabe, S. Kodambaka, W. Swiech, J.E. Greene, and D.G. Cahill, "LEEM Study of Island Decay on Si(110)," Surf. Sci. (in press).

[57] J. R. Serrano and D. G. Cahill, "Laser-induced blistering of thin $\mathrm{SiO}_{2}$ on $\mathrm{Si}$," submitted for publication.

[58] A. Raviswaran and D. G. Cahill, "Morphology of low temperature homoepitaxial growth on laser textured Ge(001)," Phys. Rev. B 69, 165313 (2004).

[59] X.-Y. Hu, D. G. Cahill, and R. S. Averback, "Dewetting and nanopattern formation of thin Pt films on $\mathrm{SiO}_{2}$ induced by ion beam irradiation," J. Appl. Phys. 89, 7777 (2001). [60] S.G. Mayr, R.S. Averback, "Evolution of morphology in nanocrystalline thin films during ion irradiation," Phys. Rev. B 68, 075419, (2003).

[61] S.G. Mayr and R.S. Averback, "Effect of ion bombardment on stress in thin films," Phys. Rev B. 68, 214105 (2003).

[62] S.G. Mayr and R.S. Averback, "Surface smoothing of rough amorphous films by irradiation-induced viscous flow," Phys. Rev. Lett. 87, 6106 (2001).

[63] D. Gall, R.T. Haasch, N. Finnegan, T.-Y. Lee, C.-S. Shin, E. Sammann, J.E. Greene, and I. Petrov, "In-situ X-ray Photoelectron, Ultraviolet Photoelectron, and Auger Electron Spectroscopy Spectra from First Row Transition-Metal Nitrides: ScN, TiN, VN, and CrN," Surf. Sci. Spectra 7,167 (2000).

[64] D. Gall, I. Petrov, and J. E. Greene, "Epitaxial Sc1-xTixN(001): Optical and Electronic Transport Properties," J. Appl. Phys. 89, 401 (2001).

[65] D. Gall, M. Städele, K. Järrendahl, I. Petrov, P. Desjardins, R.T. Haasch, T.-Y. Lee, and J.E. Greene, "Electronic Structure of ScN Determined using Optical Spectroscopy, Photoemission, and ab initio Calculations," Phys. Rev. B 63, 125119 (2001). [66] J.-S. Chun, P. Desjardins, C. Lavoie, I. Petrov, C. Cabral, Jr., and J.E. Greene, "Interfacial Reaction Pathways and Kinetics During Annealing of 111-Textured Al/TiN 
Bilayers: A Synchrotron X-Ray Diffraction and Transmission Electron Microscopy Study," J. Vac. Sci. Technol. A 19, 2207 (2001).

[67] D. Gall, M. Stoehr, and J.E. Greene, "Vibrational Modes in Epitaxial Ti1-x $S c_{x} N(001)$ Layers: An ab-initio Calculation and Raman Spectroscopy Study," Phys. Rev. B 64, 174302 (2001).

[68] D. Gall, C.-S. Shin, R.T. Haasch, I. Petrov, and J. E. Greene, "Band Gap in Epitaxial NaClstructure CrN(001) Layers," J. Appl. Phys. 91, 5882 (2002).

[69] C.-S. Shin, D. Gall, Y.-W. Kim, N. Hellgren, I. Petrov, and J.E. Greene, "Development of Preferred Orientation in Polycrystalline NaCl-structure $\delta$-TaN Layers Grown by Reactive Magnetron Sputtering: Role of Low-Energy Ion/Surface Interactions," J. Appl. Phys. 92, 5084 (2002).

[70] C.-S. Shin, D. Gall, N. Hellgren, J. Patscheider, I. Petrov, and J. E. Greene, "Vacancy Hardening in Single-crystal TiNx(001) Layers," J. Appl. Phys. 93, 6024 (2003). [71] T.-Y. Lee, D. Gall, C.-S. Shin, N. Hellgren, I. Petrov, and J. E. Greene, "Growth and Physical Properties of Epitaxial CeN Layers on MgO(001), J. Appl. Phys. 94, 921 (2003). [72] H.-S. Seo, T.-Y. Lee, J. G. Wen, I. Petrov, and J. E. Greene, "Growth and Physical Properties of Epitaxial HfN Layers on MgO(001)," J. Appl. Phys. 96, 878 (2004).

[73] T. -Y. Lee, S. Kodambaka, J. G. Wen, R. Twesten, J. E. Greene, and I. Petrov, "Controlled Nanostructural Evolution in Ti0.8Ce0.2N Layers Grown on $\mathrm{SiO}_{2}$ by Magnetron Sputter Deposition as a Function of Low Energy, High Flux Ion Irradiation," Appl. Phys. Lett. 84, 2796 (2004).

\section{Functional Nanoscale Materials and Systems}

\section{Task 1: Supramolecular Engineering of Multifunctional Materials}

Primary Participants: P. V. Braun, M. S. Gin, J. S. Moore, R. G. Nuzzo, K. S. Schweizer, K. S. Suslick, S. C. Zimmerman

\section{Task 2: Multiscale Assembly and Function}

Primary Participants: P. V. Braun, J. A. Lewis, R. G. Nuzzo, J. A. Rogers, K. S. Schweizer, C. F. Zukoski

The research in this cluster has evolved considerably from the previous cluster entitled "Nanoscale Synthesis and Mesoscale Engineering". That program resulted in 405 publications (including 8 in Nature, 26 in J. Am. Chem. Soc. and 16 in Appl. Phys. Lett.). Among the students at all levels who participated, $85 \mathrm{Ph} . \mathrm{D}$. degrees were awarded and a similar number of postdoctoral fellows sponsored. Major activities included: the synthesis and assembly of structure-controlled molecular building blocks; structure, gelation, and crystallization of colloidal suspensions - theory and experiments; novel methods of fabricating microperiodic structures. Selected highlights taken from this work are provided below.

One of the main synthetic objectives has been the development of self-organizing and function-directed synthesis as a means to responsive nanostructures. Large, structurally well-defined molecules offer attractive platforms for rationally designing nanoscale assembles (discrete or periodic) with molecular-based functions. This research provided 
new molecular building blocks that are readily synthesized and that organize in largely predicable ways on the basis of molecular shape, conformation, and non-covalent interactions. In the examples cited below, self-assembly has been effectively used as a means to create large internal voids of controlled size and shape, yielding materials with promise for applications in sensing, catalysis, and energy storage.

In one approach (Fig. 1), a convenient, multigram-scale synthesis of arylene ethynylene macrocycles was demonstrated based on newly-developed and highly-active, alkyne metathesis catalysts ${ }^{[1]}$. Driven by the precipitation of a diarylacetylene byproduct, the alkyne metathesis produces the desired macrocycles in a single step from simple monomers in high yields. The products are obtained under thermodynamic control, and high yields can be achieved without resorting to high dilution conditions. Shapepersistent macrocycles ${ }^{[2]}$ similar to the indicated tetracarbazole have been found to organize into tubular discotic liquid crystal phases ${ }^{[3]}$. The carbazole moiety imparts interesting electroactive properties to these mesogens. The catalyst we developed ${ }^{[4]}$ is selective and robust, which has enabled the synthesis of high-molecular weight electroactive polymers such as poly(p-phenyleneethynylene) $\mathrm{s}^{[5]}$ and poly $(2,5-$ thienyleneethynylene)s . Efforts to further enhance the utility of these catalysts using surface-functionalized nanoparticles are currently underway.

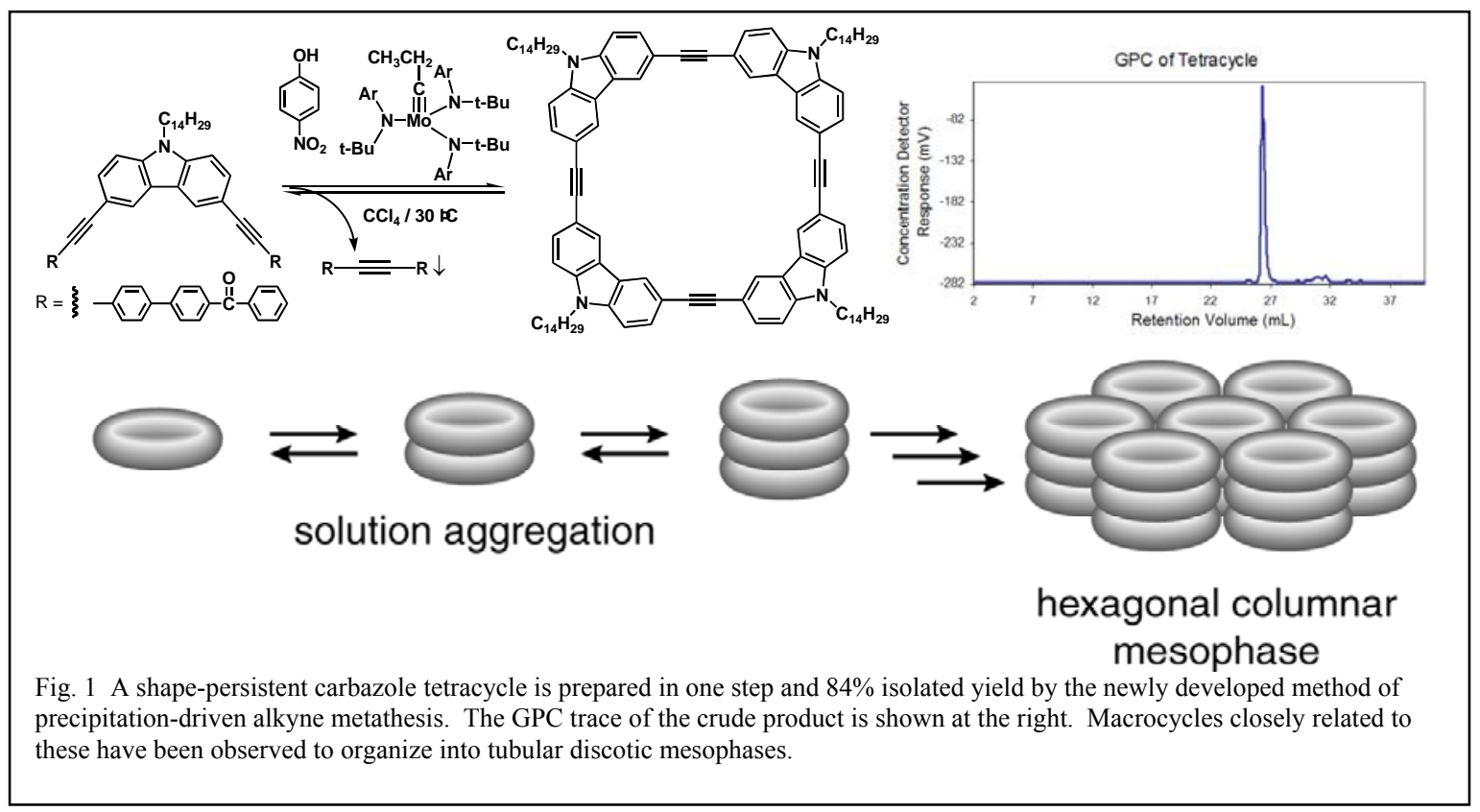


A new class of nanoporous organic solids that can separate molecules based on their size was created ${ }^{[6]}$. These organic zeolite analogues are more than 50 percent empty space - interstices that can trap molecules of the right size and shape, including water (Fig. 2) They bind water ca. hundred-times faster and two-times more strongly than the best drying agents and have a five-fold higher capacity for storing water than the best conventional zeolites. The material also shows shape selectivity, permitting only a narrow range of molecular shapes to enter.

In another approach (Fig. 3), tubular cavities have been created by the solvophobically driven helical conformation of discrete m-phenyleneethynylene

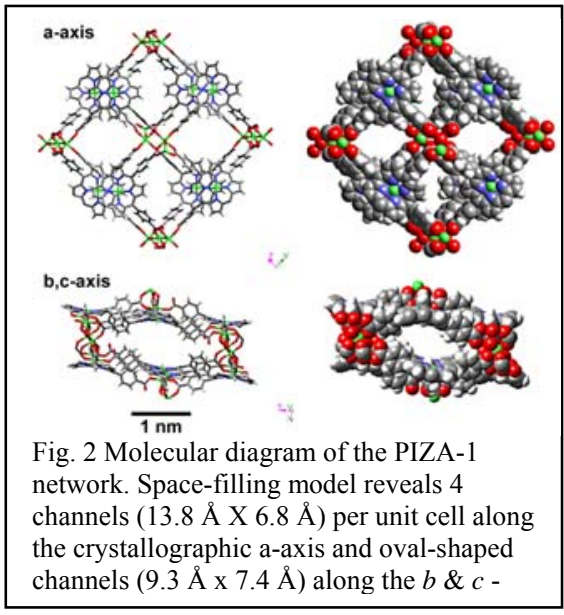
oligomers.

Systematic studies of chain length and backbone type have revealed a rich phase behavior for these oligomers in the solid state ${ }^{[7]}$. We have observed organized structures in which the helical chains pack atop

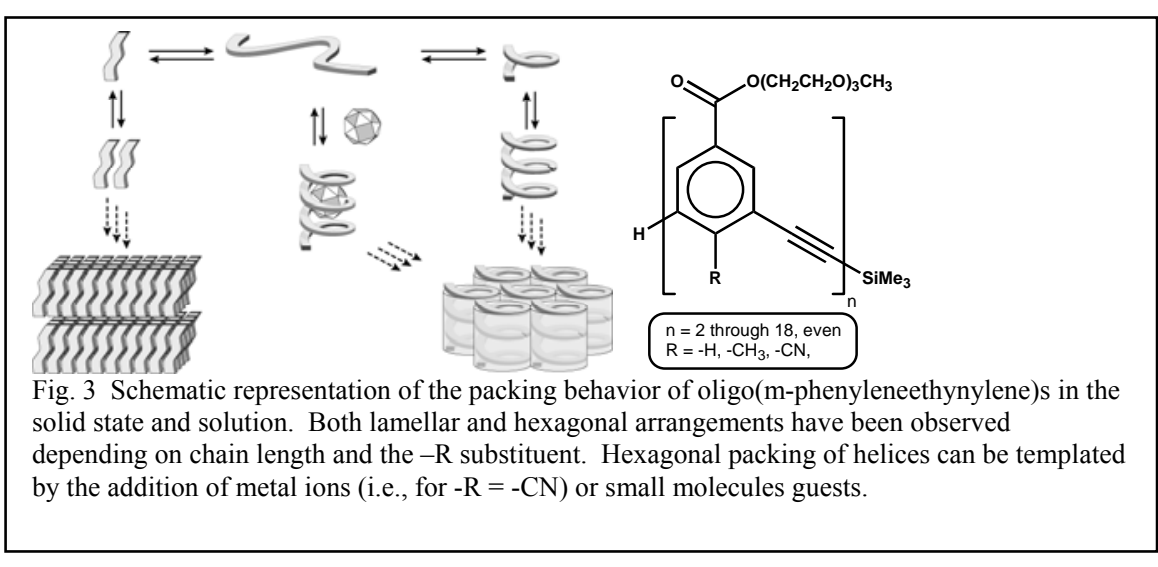
one another to create hexagonally arranged nanotubes. We have also observed lamellar arrangements in which the helical conformation unwinds and the resulting ribbon-like strands pack side-by-side. Interestingly, it has been possible to transform the lamellar arrangement into the helical arrangement by a template effect in which a small-molecule or metal ion that specifically binds in the helix interior biases conformation during the assembly process. Our future plans will utilize the understanding we have gained to develop systems that can be reversibly triggered by external chemical or physical stimuli between conformationally ordered and disorder forms. By coupling this conformational change to a self-assembly process, we will be able to engineer highly cooperative behavior triggered by external stimuli. These materials may yield new forms of actuators for application in MEMS and sensing.

We were the first group to demonstrate the creation of $3 \mathrm{D}$ waveguide structures in selfassembled photonic crystals (Fig. $4 a)^{[8]}$. This work utilized multiphoton polymerization to create optically active features inside of a photonic crystal. This advance is enabling both

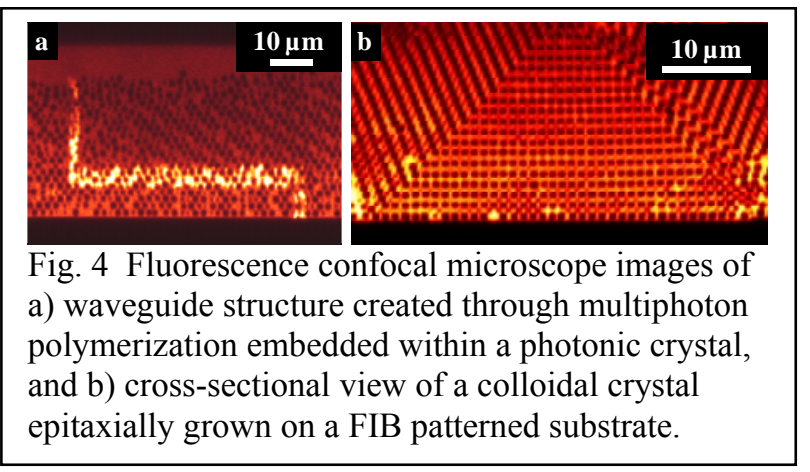


new basic science and the creation of a wide range of optical devices inside of selfassembled materials. A shortcoming of this approach, however, was that the structures had low refractive index contrasts (being composed of low refractive index materials such as air, polymer, and $\mathrm{SiO}_{2}$ ). We recently demonstrated, however, the infilling of the interstitial spaces of colloidal crystals with $\mathrm{Si}$, a high refractive index material, and will extend these approaches to enable the direct replication of photonic crystals containing embedded polymeric structures, procedures that will yield true 3D photonic bandgap devices. As part of our efforts to self-assemble defect free photonic crystals, we demonstrated the epitaxial growth of colloidal crystals on pattered substrates from binary fluids containing both nanoparticles and colloidal microspheres mixtures (Fig. $4 \mathrm{~b})^{[9]}$. The large area patterning of defect free colloidal crystals is necessary for their use in optical applications. Over the next few years, we intend to explore new routes to the formation of photonic crystals, ones based on hydrogen bond driven assembly of colloids and thermally responsive dense colloidal suspensions. Creation of large area photonic crystals with low defect density and defined orientation will be critical for the realization of technologies using self-assembled photonic devices. Such structures are of great interest for sensing applications and may hold value for contexts related to Homeland Security.

The exploitation of structures derived from complex assembly processes has benefited from critical new understandings of materials theory developed by the cluster. In a notable advance, a novel microscopic statistical mechanical theory of the structure, thermodynamics, and equilibrium phase behavior of suspensions of spherical colloids or nanoparticles mixed with nonadsorbing flexible polymers has been developed ${ }^{[10]}$. Macromolecule conformational entropy, excluded volume interactions and solvent quality have all been taken into account. The theory predicts polymer-induced depletion attractions result in a major structural reorganization of the particles on local, mesoscopic and global length scales which depend sensitively on polymer concentration, polymer size, and particle volume fraction. The structural correlations ${ }^{[11,12]}$, thermodynamic properties $^{[13]}$, and phase separation ${ }^{[14,15]}$ predictions have been verified by USAXS and related experiments carried out at the Advanced Photon Source at Argonne National Laboratory. The equilibrium theory has been combined with statistical dynamical ideas to construct a quantitative description of the nonequilibrium fluidto-gel transition and gel elasticity ${ }^{[16]}$.

The theory predicts (Fig. 5) that the tendency for the suspension to gel is monotonically enhanced as the polymer-to-particle size asymmetry ratio, $R_{g} / R$, decreases. The shape of the gelation boundaries is of a power law form at low and moderate volume fractions, and changes to a stronger exponential dependence in concentrated suspensions. The gel elastic shear modulus is predicted to depend in a distinctive power law fashion on polymer concentration, size asymmetry ratio and particle volume fraction. All the theoretical results have been

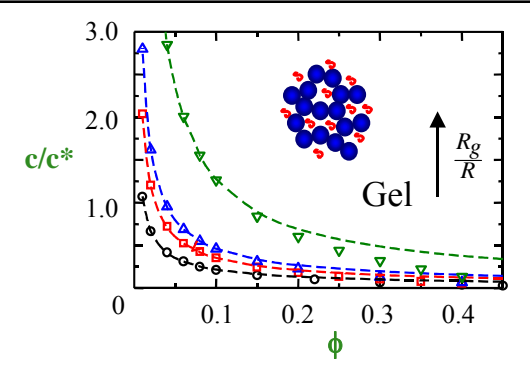

Fig. 5 Theoretical predictions for the fluid to gel nonequilibrium transition boundaries of polymer-nanoparticle suspensions in the reduced polymer concentration-particle volume fraction plane. The gelation boundary moves up with increasing polymer to particle size ratio $\mathrm{R}_{\mathrm{g}} / \mathrm{R}=0.025,0.06,0.090 .37$. The dashed lines indicate power law behavior. 
verified by the experiments on silica-polystyrene suspensions and recent measurements of others on buoyancy matched systems. Hence, accurate design rules for controlling gelation and soft elasticity via manipulation of multiple material variables have now been established.

We have developed a fundamental understanding of the phase behavior, structure, rheology, and assembly of binary mixtures comprised of polyelectrolyte complexes ${ }^{[17]}$ and colloidal microsphere-nanoparticle mixtures. These binary mixtures exhibit rich and useful phase behaviors that are driven by both entropic and enthalpic considerations. To systematically investigate their behavior and properties, we have employed a myriad of analytical techniques housed in the Center for Microanalysis of Materials (CMM) as well as direct imaging by confocal microscopy and shear viscometry/oscillatory measurements. We have exploited the unique attributes of these binary mixtures to create 3D microperiodic materials with submicron features that may find application as photonic band gap materials, advanced sensors, or novel scaffolds. Specifically, we have demonstrated the use of concentrated polyelectrolyte complexes as inks for the direct writing of 3D micro-periodic structures, as shown in Fig. $6^{[18,19]}$. We have further demonstrated the nanoparticlemediated epitaxial assembly of colloidal crystals with defined crystallographic orientation, low defect density, and the mechanical robustness required for these targeted applications (Fig. 4) ${ }^{[9]}$. Our prior research accomplishments have provided an

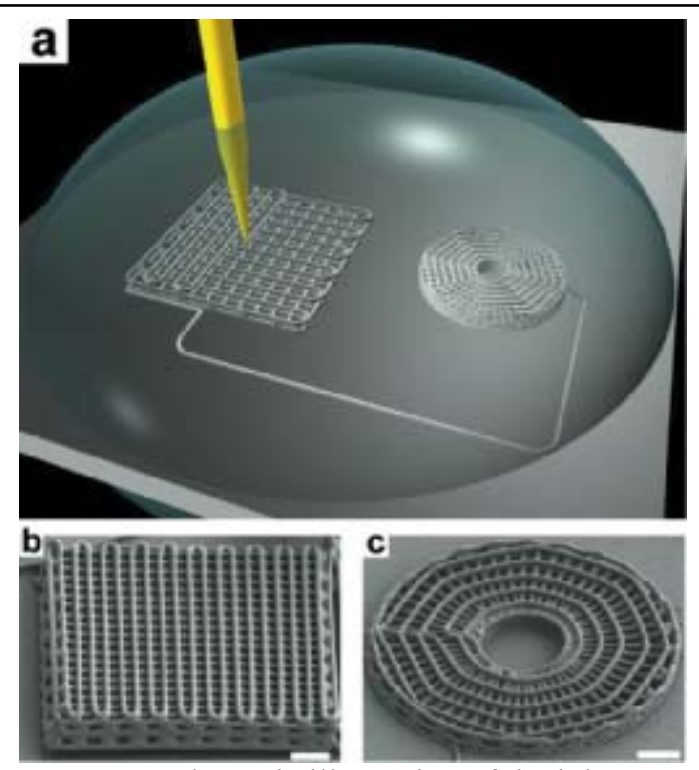

Fig. 6. a, Schematic illustration of the ink deposition process (not drawn to scale). A concentrated polyelectrolyte ink is housed in the syringe (shown in yellow) immersed in a coagulation reservoir (grey hemispherical drop) and deposited onto a glass substrate (shown in light grey). b, 3-D periodic structure with a face-centered tetragonal geometry (filament diameter $=1 \mu \mathrm{m}, 10$ layers). c, 3-D radial array (filament diameter $=1 \mu \mathrm{m}, 5$ layers). essential platform for the future activities outlined below, which build directly upon these efforts as well as greatly extend our capabilities and fundamental understanding into new and critically important directions for the DOE-MRL program. These results mirror the progress made in the areas of nanoscale materials patterning and fabrication more generally during the past review period. These are world-leading competencies unique to the collaborating team and serve as a strong foundation on which to build the ambituous new programmatic efforts described below.

\section{Selected Publications:}

[1] W. Zhang and J. S. Moore, "Arylene Ethynylene Macrocycles Prepared by Precipitation-Driven Alkyne Metathesis," J. Am. Chem. Soc. 126, 12796 (2004). [2] D. H. Zhao and J. S. Moore, "Shape-Persistene Arylene Ethynylene Macrocycles: 
Syntheses and Supramolecular Chemistry," Chem. Commun. 807-818 (2003). [3] O. Y. Mindyuk, M. R. Stetzer, P. A. Heiney, J. C. Nelson and J. S. Moore, "HighResolution X-ray Diffraction Study of a Tubular Liquid Crystal," Adv. Mater. 10, 13631366 (1998).

[4] W. Zhang, S. Kraft and J. S. Moore, "Highly Active Trialkoxymolybdenum(VI) Alkylidyne Catalysts Synthesized by a Reductive Recycle Strategy," J. Am. Chem. Soc. 126, 329-335 (2004).

[5] W. Zhang and J. S. Moore, "Synthesis of Poly(2,5-thienyleneethynylene)s by Alkyne Metathesis," Macromolecules 37, 3973-3975 (2004).

[6] M. E. Kosal, J. H. Chou, S. R. Wilson and K. S. Suslick, "A Functional Zeolite Analogue Assembled from Metalloporphyrins," Nature Mater. 1, 118-121 (2002).

[7] C. Kubel, M. J. Mio, J. S. Moore and D. C. Martin, "Molecular Packing and Morphology of Oligo(m-phenylene ethynylene) Foldamers," J. Am. Chem. Soc. 124, 8605-8610 (2002).

[8] W. Lee, S. A. Pruzinsky and P. V. Braun, "Multi-photon Polymerization of Waveguide Structures Within Three-Dimensional Photonic Crystals," Adv. Mater. 14, 271-274 (2002).

[9] W. Lee, A. Chan, J. A. Lewis and P. V. Braun, "Nanoparticle-Mediated Epitaxial Assembly of Colloidal Microspheres on Patterned Substrates," Langmuir 20, 5262-5270 (2004).

[10] M. Fuchs and K. S. Schweizer, "Structure of Polymer-Colloid Suspensions," J. Physics-Condensed Matter 14, R239 (2002).

[11] A. Shah, S. Ramakrishnan, Y. L. Chen, K. S. Schweizer and C. F. Zukoski, "Small Angle Scattering Studies of Colloid-Polymer Suspensions," Langmuir 19, 5128 (2003). [12] A. Shah, S. Ramakrishnan, Y. L. Chen, K. S. Schweizer and C. F. Zukoski, "Microstructure of Dense Colloid-Polymer Suspensions and Gels," J. Physics-Condensed Matter 15, 4751 (2003).

[13] S. Ramakrishnan, M. Fuchs, K. S. Schweizer and C. F. Zukoski, "Concentration Fluctuations in a Model Colloid-Polymer Suspension: Experimental Tests of Depletion Theories," Langmuir 18, 1082 (2002).

[14] S. Ramakrishnan, M. Fuchs, K. S. Schweizer and C. F. Zukoski, "Entropy Driven Phase Transitions in Colloid-Polymer Suspensions: Tests of Depletion Theories," J. Chem. Phys. 116, 2211 (2002).

[15] A. Shah, Y. L. Chen, K. S. Schweizer and C. F. Zukoski, "Phase Separation and Concentration Fluctuations in Polymer-Colloid Mixtures Under Near Theta Solvent Conditions," J. Chem. Phys. 118, 3350 (2003).

[16] Y. L. Chen and K. S. Schweizer, "Theory of Gelation and Elasticity in PolymerParticle Suspensions," J. Chem. Phys. 120, 7212 (2004).

[17] G. M. Gratson and J. A. Lewis, "Polyelectrolyte Inks for Direct-Write Assembly of 3-D Micro-Periodic Scaffolds," Langmuir In Press, (2004).

[18] G. M. Gratson, M. J. Xu and J. A. Lewis, "Direct Writing of Three-Dimensional Webs," Nature 428, 386 (2004).

[19] J. A. Lewis and G. M. Gratson, "Direct Writing in Three Dimensions," Materials Today July/August, 32-39 (2004). 


\section{Quantum Materials and Nanodevices}

Task 1: Quantum dynamics and decoherence in nanoscale devices: quantum dots, wires, junctions, and arrays

Primary Participants: A. Bezryadin, R. Budakian, P.M. Goldbart, A. J. Leggett, N. Makri, R.M. Martin, N. Mason, M.B. Salamon, and D.J. Van Harlingen

\section{Task 2: Highly tunable states of complex quantum materials}

Primary Participants: S.L. Cooper, L.H. Greene, P.M. Goldbart, A.J. Leggett, R.M. Martin, N. Mason, M.B. Salamon, D.J. Van Harlingen

The cluster research has resulted in 365 primary publications, including 7 in Science or Nature, and 46 in Physical Review Letters or Applied Physics Letters. 50 Ph.D. degrees were awarded to participating graduate students. A more detailed overview of the progress made during the most recent review period is provided by the summary materials given in the later sections of this review document.

\section{Complex oxide materials: Competing states and colossal responses}

Salamon's work has explored the behavior of doped $\mathrm{LaMnO}_{3}$ and the low temperature behavior of unconventional superconductors. While colossal magnetoresistance has been the hallmark of $\mathrm{La}_{1-\mathrm{x}} \mathrm{Ca}_{\mathrm{x}} \mathrm{MnO}_{3}$ and related materials, the overall magnetic properties are also significantly different from conventional ferromagnets. In samples with $x=1 / 3$, Salamon's group showed ${ }^{[1]}$ that the thermodynamics of these complex oxides should be treated in the context of a Griffiths phase, in which magnetic order arises via the propagation of numerous correlated clusters rather than via the divergence of a ferromagnetic correlation length. On the $x=2 / 3$ end of the phase diagram, Salamon discovered, along with $\mathbf{Z u o}^{[2]}$, that orthorhombic, ferromagnetic regions grow after quenching through the charge-ordering transition. It was found that a quantal-phase interpretation of the anomalous Hall effect, developed with Goldbart, holds for a simple ferromagnet such as $\mathrm{Gd}^{[3]}$. Ongoing work on manganites by Goldbart's group, which builds on earlier work with Salamon, aims to develop a comprehensive theory of the phase diagram ${ }^{[4]}$, charge transport ${ }^{[5]}$, magnetic defect structures ${ }^{[6]}$ in these rich and subtle systems.

During the past several years, Cooper's group has been using various optical spectroscopic techniques, along with high pressure, high magnetic field, and low temperature methods, to explore a number of scientifically and technologically interesting phenomena in materials exhibiting complex quantum-mechanical behaviors. These include exotic phase transformations; self-organization of charges, orbitals, and/or spins; nanoscale electronic and magnetic phase inhomogeneity; and "colossal" sensitivities of structural, magnetic, and electronic properties to external perturbation. For example, using temperature- and field-dependent optical spectroscopic methods, Cooper's group demonstrated that both metallic ferromagnetism and colossal magnetoresistance in ferromagnetic semiconductors (e.g., manganese perovskites ${ }^{[7]}$ and Eu-based materials ${ }^{[8]}$ ) are precipitated by the formation, evolution, and eventual percolation of ferromagnetic clusters. Various optical spectroscopic methods have also 
been used by Cooper's group to study the self-organization of charge through the "charge-ordering" phase transition of manganese perovskites ${ }^{[9,10]}$. These studies revealed that long-range charge order is precipitated by the development of charge-stripe fragments that evolve and eventually percolate below the charge-ordering temperature.

Using a novel apparatus for performing optical spectroscopy at high pressures and low temperatures, the evolution and eventual melting of the charge density wave state $(\mathrm{CDW})$ in $1 \mathrm{~T}-\mathrm{TiSe}_{2}$ was also studied ${ }^{[11]}$ by Cooper's group, in collaboration with Chiang. This investigation revealed that pressure-induced "quantum" melting transition of the charge density wave state involves an interesting intermediate phase regime characterized by local CDW order, but no long-range CDW order. The results were well correlated with the electronic structure determined by Chiang's group using angleresolved photoemission.

Cooper's group also used pressure-tuned optical spectroscopy to study the pressure-induced collapse of both orbital-order and the antiferromagnetic ground state of layered ruthenate materials, demonstrating the intimate connection between orbital order and antiferromagnetism ${ }^{[12]}$; see Fig. 1. More recent field-dependent optical measurements by Cooper's group have further shown that a magnetic field can be used to alter the orbital population, and hence the electron mobility. These studies demonstrate that, by exploiting the strong spin-orbit coupling in these materials, the electronic properties can be sensitively tuned by controlling the spin orientation of the ruthenium moments ${ }^{[13]}$.

Martin's research is focused upon the development of computational methods and their application to electronic phenomena exhibited in complex systems. His exemplary calculations of the electronic properties of $\mathrm{C}_{28}$-derived doped fulleride solids (see Fig. 2) led to the prediction of stable structures with superconducting transition temperatures estimated to be $\sim 60 \mathrm{~K}^{[14]}$. Martin is the author of a recent book ${ }^{[15]}$, which is the most extensive exposition available of the concepts, theory and computational methods for electronic structure of materials. His group's work was recognized recently when a DOE-supported student Ivo Souza was awarded the 2004 George E. Valley, Jr. Prize from the American Physical Society for "fundamental advances in the theory of polarization, localization and electric fields in crystalline

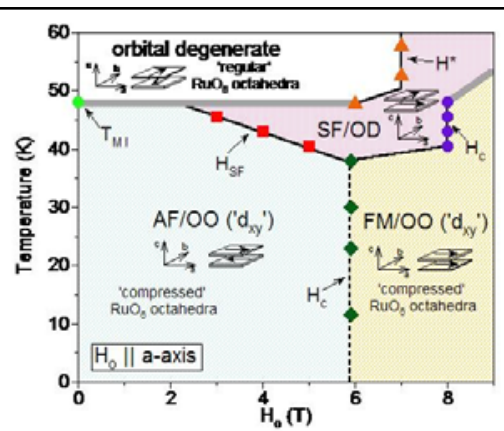

Fig. 1. Magnetic field vs. temperature phase diagram for orbital, magnetic, and conducting phases of $\mathrm{Ca}_{3} \mathrm{Ru}_{2} \mathrm{O}_{7}$, deduced from light scattering measurements of magnon and phonon energies. Shown are antiferromagnetic (AF), orbitalordered $(\mathrm{OO})$, orbital-disordered (OD)spin-flop (SF) and phases and the boundaries between them.

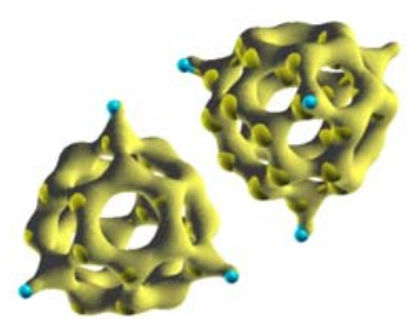

Fig. 2. Isocharge density surface (0.2 a.u.) of hyperdiamond $\mathrm{C}_{28} \mathrm{H}_{4}$ solid. Notice that the solid is held by weak bonds as evidenced by the lack of charge between the constituent molecular units. insulators." 


\section{Superconductivity in oxides and other novel and unconventional materials}

Salamon's work has focused on the lowtemperature behavior of unconventional superconductors. Continuing a long-standing project on the low-temperature penetration depth in collaboration with Van Harlingen, Salamon's group has found strong evidence ${ }^{[16]}$ for a longpredicted spontaneous vortex phase in the weakly ferromagnetic superconductor $\mathrm{ErNi}_{2} \mathrm{~B}_{2} \mathrm{C}$. This phase manifests itself via an increasing penetration depth as the temperature is reduced below the ferromagnetic transition at $2.4 \mathrm{~K}$. At still lower temperatures, pinning causes a sharp decrease in the penetration depth to its low temperature value. As shown in Fig. 3, the penetration depth of the filled skutterudite superconductors $\mathrm{PrMSb}_{12}$ (where $\mathrm{M}$ is a mixture of $\mathrm{Os}$ and $\mathrm{Ru}$ ) has been measured. On the pure Os end, the material is a heavy fermion metal that exhibits point nodes in its gap function ${ }^{[17]}$. On the $\mathrm{Ru}$ rich side ${ }^{[18]}$, and

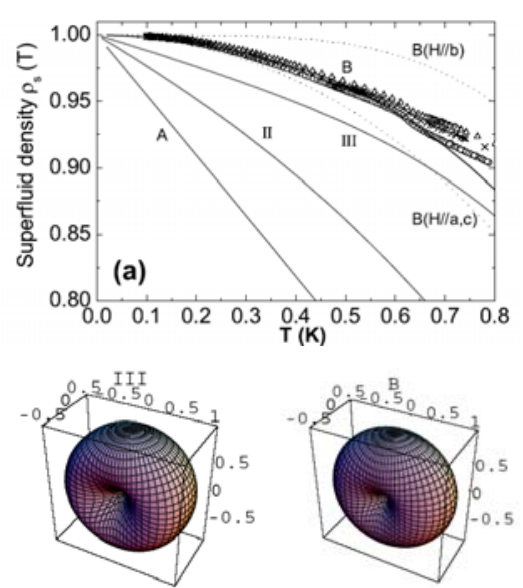

Fig. 3. The density of superconducting electrons in the skutterudite compound $\mathrm{PrOs}_{4} \mathrm{Sb}_{12}$. The gap functions B and III, look similar but only $\mathrm{B}$, which exhibits time-reversal-symmetry breaking, is capable of fitting the data [18]. indeed for $\mathrm{Ru}$ content greater than $30 \%$, the material is a conventional BCS superconductor ${ }^{[19]}$. There is a phase transition line between conventional and nodal superconductivity that appears to end in a quantum critical point near.

Van Harlingen's principal effort has been to understand the wealth of new and exciting phenomena that arise in complex oxide superconductors as a result of the strong electronic correlations and the competition between Coulomb and magnetic interactions. His approach has been to develop and apply novel experimental techniques and local electronic and magnetic probes sensitive to the nanoscale lengths relevant to these phenomena. Over the last few years, the emphasis has been on elucidating the symmetry and mechanism of unconventional superconductors, with particular attention given to the possibility of complex order parameter states that break time-reversal symmetry and to understanding the nature of the anomalous normal state pseudogap regime. Notable achievements coming from this work include: (i) Two-coil screening experiments in BSCCO thin films that measured the superfluid density, observing critical dynamical scaling at the superconducting transition and extracting characteristic critical exponents ${ }^{[20]}$ - these measurements show that there is no abrupt change in superfluid density as a function of doping, strong evidence against the $T^{*}$ pseudogap crossover line cutting through the superconducting dome, behaviors suggested by quantum critical models; (ii) Grain boundary interferometry measurements on cuprates, which tested for the onset of subdominant complex superconducting phases induced by the d-wave order parameter at surfaces ${ }^{[21]}$, showing instead that the $\mathrm{d}$-wave state is remarkably robust; and (iii) Transport measurements on nanowires of underdoped cuprates, which reveal anomalous domain switching fluctuations above the transition temperature, providing possible evidence for charge stripe domain dynamics in the pseudogap state ${ }^{[22]}$. 
Greene's work has focused on point contact spectroscopy studies of novel and unconventional superconductors and the Andreev conversion process between conventional superconductor and heavyfermion metals. She has designed and built a state of the art mechanical tunneling apparatus (see Fig. 4) to measure the electronic structure of novel superconducting materials at temperatures down to $300 \mathrm{mK}$ and applied magnetic fields up to $12 \mathrm{~T}$. This system uses a piezoelectric bimorph cantilever to achieve precise control of the separation between tunneling electrodes. It has been used in two modes to probe the density of states: (i) mechanical planar tunneling (MPT); and (ii) point-contact spectroscopy $(\mathrm{PCS})^{[23]}$, in which Andreev reflection processes dominate ${ }^{[24]}$. She has achieved high reproducibility in the PCS mode with single crystals of the newlydiscovered heavy-fermion superconductor $\mathrm{CeCoIn}_{5}$ in collaboration with J. L. Sarrao and J. D. Thompson (Los Alamos National Laboratory) ${ }^{[25]}$ (see Fig. 5), and $\mathrm{MgB}_{2}$ thin films, which exhibit two distinct energy gaps.

Greene's group is studying $\mathrm{CeCoIn}_{5}$ $\left(\mathrm{T}_{\mathrm{c}}=2.3 \mathrm{~K}\right)$ because this system exhibits a magnetic ground state and is proximate to an antiferromagnetic quantum critical point, possibly associated with the FuldeFerrel-Larkin-Ovchinnikov (FFLO) phase transition $^{[26,27]}$. There is considerable thermodynamic evidence that the pairing symmetry of this superconductor is dwave, and this is supported by spectroscopic data ${ }^{[25]}$. An extended Blonder-Tinkham-Klapwijk (BTK) ${ }^{[28]}$ model invoking a d-wave superconducting order parameter ${ }^{[29]}$ best fits the zero-bias conductance data. To date, this is the only spectroscopic evidence that $\mathrm{CeCoIn}_{5}$ is a dwave, strongly-coupled superconductor $\left[2 \Delta(0) / \mathrm{k}_{\mathrm{B}} \mathrm{T}_{\mathrm{c}}=4.6\right]$. An enhanced subgap conductance is shown to arise from the Andreev reflection process, a result that is extremely intriguing because standard theories predict that tunneling into heavy fermion superconductors should not yield Andreev reflections at all, due to their extreme mass- 
mismatch. Greene has quantified the Andreev reflection signal, finding it to be reduced by an order of magnitude from that in conventional superconductors, but the nature of the Andreev conversion process at the heavy fermion interface remains an open question.

Work in Leggett's group includes an investigation of Andreev reflection from the interface between a normal metal and a heavy-fermion superconductor. This work was stimulated by Greene's experiments on current transmission through the interface between $\mathrm{Au}$ and the heavy-fermion superconductor $\mathrm{CeCoIn}_{5}$, which apparently cannot be explained in terms of any standard model. In related work, Leggett's group has been studying charge-carrier transmission through interfaces between superconductor and quantum Hall effect regions ${ }^{[30]}$.

Goldbart's group has also continued its investigations of low-energy electronic states associated with impurities in unconventional superconductors. They have shown how STM measurements in the pseudogap regime are capable of detecting signatures of local superconductivity, if it is present, through a fluctuation-broadened peak in the low-energy density of states ${ }^{[31]}$, and they have explored the formation of a low-energy impurity band in d-wave superconductors, due to the hybridization of low-energy impurity states ${ }^{[32]}$.

\section{Quantum properties of nanoscale devices}

Goldbart's group has explored the theme of charge transport in a range of settings, including inhomogeneous magnetic and superconducting systems, as well as carbon nanotubes and nanoscale superconducting devices. In collaboration with Bezryadin's group, Goldbart's group showed, experimentally and theoretically, that carbon nanotubes should undergo a striking inter-conversion between metallic and insulating behavior, modulated by the magnetic flux threading the tube $\left[{ }^{32}\right]$; see Figs. 6 and 7.

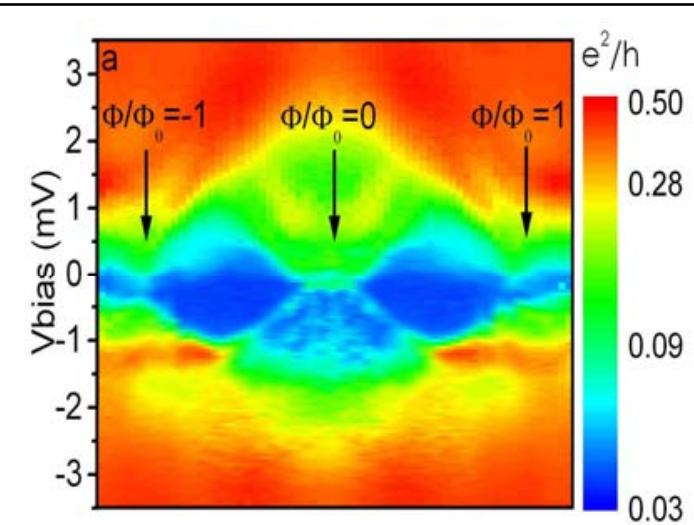

Fig. 7. "Conductance map" vs. magnetic field for a single-electron-tunneling transistor made of a multiwalled carbon nanotube threaded by magnetic flux [32].

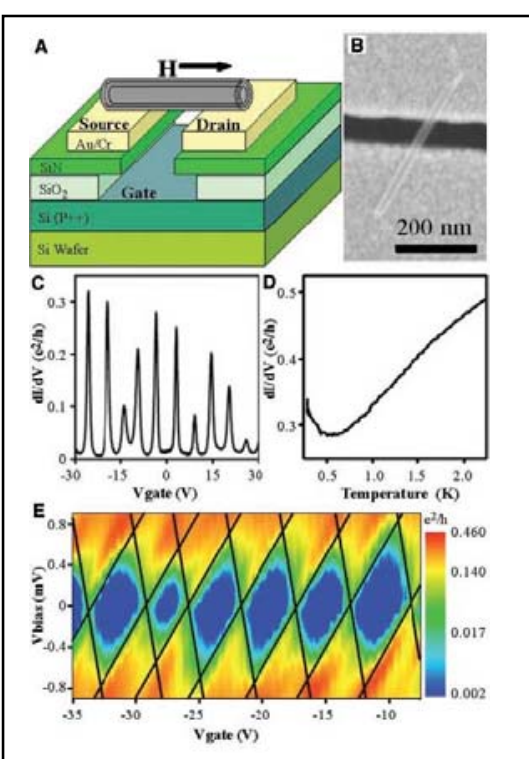

Fig. 6. Fabrication of and measurements on a single-electron transistor built from a gated multi-walled carbon nanotube. A: Schematic of the device. B:

Scanning electron micrograph showing a nanotube straddling a narrow trench. C: Coulomb blockade peaks, showing that the nanotube is acting as a quantum dot. D: Temperature dependence of a peak height, suggesting transport via resonant tunneling. E: Coulomb blockade diamonds. 
More recently, these two groups have devised and performed experiments on a superconducting phase gradiometer device ${ }^{[33]}$. This device consists of a pair of superconducting films, connected in parallel by a pair of ultra-narrow superconducting bridges, fabricated by depositing metal on two DNA template molecules connecting the films. It is a phase gradiometer in the sense that it is sensitive to spatial variations in the phase of the superconducting order in the leads. Magnetoresistance experiments on this device reveal striking oscillations, whose origin, period and amplitude have been determined via a detailed theoretical picture that the groups have developed ${ }^{[34]}$. In the setting of inhomogeneous superconducting systems, Goldbart's group has introduced and developed the theory of Andreev billiards - normal metal arenas for quasiparticle motion, surrounded by superconductor, which confines the quasiparticles and induces a novel form of chaotic motion in them ${ }^{[35]}$.

Martin's group has successfully predicted the structures of self-assembled Au nanowires on the stepped $\mathrm{Si}$ (557) surface ${ }^{[36]}$, these predictions being verified by subsequent experiments by Robinson ${ }^{[37]}$. This prediction has elicited experimental attention by many research groups. Martin's recent work has shown that the $\mathrm{Au}$ nanowires form an interesting system with large coupling of spin and charge currents ${ }^{[38]}$.

\section{Characterizing model quantum systems}

Characterizing the states of quantum systems has been explored by Goldbart's group, which has developed a robust and general scheme for quantifying quantum entanglement ${ }^{[39]}$. They have successfully applied this scheme to a variety of few- and many-particle quantum states, and have used their scheme to shed new light on quantum phase transitions ${ }^{[40]}$.

Van Harlingen's group has been undertaking studies of microscopic sources of decoherence in superconducting flux qubits for quantum computing, focusing on $1 / \mathrm{f}$ critical current noise arising from charge trapping at defect sites in the junction barrier that introduce phase noise and two-level fluctuators that entangle the qubit, causing energy level splitting ${ }^{[41]}$.

The Leggett group's work includes an investigation of the coexistence of the Mottinsulator and superfluid phases of ultracold atomic Bose gases in an optical lattice. In particular, the dependence of the coexistence curve on the temperature and the rate of inter-site tunneling is being explored. Also being investigated are kinetic aspects of the Mott-insulator to superfluid transition of bosons in an optical lattice, with particular emphasis on the time it takes to realize Bose-Einstein condensation (BEC) once the barrier heights have been reduced to the point where this condensation is energetically stable. Leggett has also been investigating the thermodynamics of the BEC-BCS crossover, as well as the expected behavior of the NMR signal.

\section{Selected Publications:}

[1] M.B. Salamon and S.H. Chun, "Griffiths singularities and magnetoresistive manganites," Phys. Rev. B 68, 014411 (2003).

[2] J. Tao, D. Niebieskikwiat, M.B. Salamon and J.M. Zuo, "Lamellar phase separation and dynamic competition in $\mathrm{La}_{0.23} \mathrm{Ca}_{0.77} \mathrm{MnO}_{3}$." Phys. Rev. B. (in press). 
[3] S. Baily and M. B. Salamon, "Berry's phase contribution to the anomalous Hall effect of gadolinium," Phys. Rev. B (in press).

[4] D. Pekker, S. Mukhopadhyay, N. Trivedi and P.M. Goldbart, "Double-exchange model: Analytical zero-temperature phase diagram," to be submitted to Phys. Rev. B.

[5] Y. Lyanda-Geller, I.L. Aleiner and P.M. Goldbart, "Anomalous Hall effect in a smooth random potential," to be submitted to Phys. Rev. B; "Magnetization inhomogeneities and anomalous Hall effect in metallic magnets," to be submitted to Phys. Rev. B.

[6] D. Pekker, P.M. Goldbart and A.G. Abanov, "Hedgehog excitations in doubleexchange magnetism: Energetics and electronic structure," to be submitted to Phys. Rev. B.

[7] S. Yoon, H.L. Liu, G. Schollerer, S.L. Cooper, P.D. Han, D.A. Payne and S.-W. Cheong, "Raman and optical spectroscopy studies of small-to-large polaron crossover in the perovskite manganese oxides," Phys. Rev. B 58, 2795 (1998).

[8] H. Rho, C.S. Snow, S.L. Cooper, Z. Fisk, A. Comment and J.-Ph. Ansermet, "Evolution of magnetic polarons and spin-carrier interactions through the metal-insulator transition in $\mathrm{Eu}_{1-\mathrm{x}} \mathrm{Gd}_{\mathrm{x}} \mathrm{O}$," Phys. Rev. Lett. 88, 127401 (2002).

[9] H.L. Liu, S.L. Cooper and S-W. Cheong, "Optical study of the evolution of charge and spin ordering in the manganese perovskite $\mathrm{Bi}_{1-\mathrm{x}} \mathrm{Ca}_{\mathrm{x}} \mathrm{MnO}_{3}$ (x > 0.5)," Phys. Rev. Lett. 81, 4684-4687 (1998).

[10] M. Ruebhausen, S. Yoon, S.L. Cooper, K.H. Kim and S.-W. Cheong, "Anisotropic optical signatures of orbital and charge ordering in $\mathrm{Bi}_{1-\mathrm{x}} \mathrm{Ca}_{\mathrm{x}} \mathrm{MnO}_{3}$," Phys. Rev. B 62, R4782 (2000).

[11] C.S. Snow, J.F. Karpus, S.L. Cooper, T.E. Kidd and T.-C. Chiang, "Quantum melting of the charge density wave state in $1 \mathrm{~T}-\mathrm{TiSe}_{2}$," Phys. Rev. Lett. 91, 136402 (2003).

[12] C.S. Snow, S.L. Cooper, G. Cao, J.E. Crow, S. Nakatsuji and Y. Maeno, "Pressuretuned collapse of the Mott-like state in $\mathrm{Ca}_{n+1} \mathrm{Ru}_{n} \mathrm{O}_{3 n+1}(\mathrm{n}=1,2)$ : Raman spectroscopic studies," Phys. Rev. Lett. 89, 226401 (2002).

[13] J.F. Karpus, R. Gupta, H. Barath, S.L. Cooper and G. Cao, "Field-induced orbital and magnetic phases in $\mathrm{Ca}_{3} \mathrm{Ru}_{2} \mathrm{O}_{7}$," Phys. Rev. Lett. 93, 167205 (2004).

[14] N.A. Romero, J. Kim and R.M. Martin, "Electron-Phonon Interactions in C28derived Molecular Solids," Phys. Rev. B 70, 140504 (2004).

[15] R.M. Martin, "Electronic Structure: Basic theory and practical methods," Cambridge University Press (2004).

[16] E.E.M. Chia, M.B. Salamon, T. Park, H.-J. Kim and Sung-ik Lee, "Observation of the spontaneous vortex phase in the weakly ferromagnetic superconductor $\mathrm{ErNi}_{2} \mathrm{~B}_{2} \mathrm{C}$ : A penetration depth study." Phys. Rev. Lett. (submitted).

[17] E.E.M. Chia, M.B. Salamon, H. Sugawara and H. Sato, "Probing the superconducting gap symmetry of $\mathrm{PrOs}_{4} \mathrm{Sb}_{12}$ : A penetration depth study," Phys. Rev. Lett. 91, 247003 (2003).

[18] E.E.M. Chia, M.B. Salamon, H. Sugawara and H. Sato, "Probing the superconducting gap symmetry of $\mathrm{PrRu}_{4} \mathrm{Sb}_{12}$ : A comparison with $\mathrm{PrOs}_{4} \mathrm{Sb}_{12}$," Phys. Rev. B 69, 180509 (2004). 
[19] E.E.M. Chia, M.B. Salamon, D. Vandervelde, D. Kikuchi, H. Sugawara and H. Sato, "Evolution of the superconducting gap in $\operatorname{Pr}\left(\mathrm{Os}_{1-\mathrm{x}} \mathrm{R}_{\mathrm{x}}\right) \mathrm{Sb}_{12}$," Phys. Rev. Lett. (submitted).

[20] K.D. Osborn, D.J. Van Harlingen, V. Aji, N.D. Goldenfeld, S. Oh, and J.N. Eckstein, "Critical Dynamics of superconducting BSCCO films", Phys. Rev. B 68, 144516 (2003).

[21] W.K. Neils and D.J. Van Harlingen. "Experimental test for subdominant superconducting phases with complex order parameters in cuprate grain boundary junctions", Phys. Rev. Lett. 88, 47001 (2001).

[22] J.A. Bonetti, D.S. Caplan, D.J. Van Harlingen, and M.B. Weissman, "Electronic Transport in Underdoped $\mathrm{YBa}_{2} \mathrm{Cu}_{3} \mathrm{O}_{7-\delta}$ Nanowires: Evidence for Fluctuating Domain Structures", Phys. Rev. Lett. 93, 087002 (2004).

[23] Y. G. Naidyuk and I.K. Yanson, "Point-contact spectroscopy of heavy-fermion systems" J. Phys.: Condens. Matter 10, 8905 (1998); H. v. Löhneysen, "Probing the energy gap of heavy-fermion superconductors" Physica B 218, 148 (1996); K. Gloos, F.B. Anders, B. Buschinger, C. Geibel, K. Heuser, F. Jährling, J.S. Kim, R. Klemens, R. Müller-Reisener, C. Schank and G.R. Stewart, "Scaling behavior of point contacts between a tungsten tip and the heavy-fermion superconductors," J. Low Temp. Phys. 105, 37 (1996); and references therein.

[24] A.F. Andreev, "The thermal conductivity of the intermediate state in superconductors,” Sov. Phys. JETP 19, 1228 (1964).

[25] W.K. Park, L.H. Greene, J.L. Sarrao and J.D. Thompson, "Point contact spectroscopy of the heavy-fermion superconductor CeCoIn 5 " (cond-mat/0409090, submitted to Phys. Rev. Lett.).

[26] See, e.g., J.D. Thompson et al., "Magnetism and unconventional superconductivity in $\mathrm{Ce}_{n} \mathrm{M}_{\mathrm{m}} \mathrm{In}_{3 \mathrm{n}+2 \mathrm{~m}}$ heavy-fermion crystals", Physica B 329-333, 446 (2003); H. Heeger et al., "Pressure-Induced Superconductivity in Quasi-2D CeRhIn 5 " Phys Rev. Lett 84, 4986 (2000); C. Petrovic et al., "Heavy-fermion superconductivity in $\mathrm{CeCoIn}_{5}$ at $2.3 \mathrm{~K}$ ", J. Phys: Cond. Mat. 13, L337-42 (2001); R. Movshovich et al., "Unconventional Superconductivity in CeIrIn 5 and $\mathrm{CeCoIn}_{5}$ : Specific Heat and Thermal Conductivity Studies" Phys Rev. Lett. 86, 5152 (2001); G. Sparn et al., "Unconventional superconductivity in CeCoIn 5 - a high pressure study," Physica B. 319, 262-7 (2002); N. Curro et al., "Anomalous NMR magnetic shifts in CeCoIn 5 ," Phys. Rev. B 64, 180514 (2001); E. E. M. Chia et al., "Nonlocality and strong coupling in the heavy fermion superconductor CeCoIn 5 : A penetration depth study," Phys. Rev. B 67, 014527 (2003).

[27] A. Bianchi, R. Movshovich, C. Capan, P.G. Pagliuso and J.L. Sarrao, "Possible Fulde-Ferrell-Larkin-Ovchinnikov Superconducting State in CeCoIn 5 ", Phys. Rev. Lett. 91, 187004 1-4 (2003).

[28] G.E. Blonder, M. Tinkham and T.M. Klapwijk, "Transition from metallic to tunneling regimes in superconducting microconstrictions: Excess current, charge imbalance, and supercurrent conversion," Phys. Rev. B 25, 4515 (1982); G.E. Blonder and M. Tinkham, "Metallic to tunneling transition in $\mathrm{Cu}-\mathrm{Nb}$ point contacts," ibid. 27, 112 (1983); G.E. Blonder, Ph.D. thesis, Harvard University, 1982.

[29] Y. Tanaka and S. Kashiwaya, "Theory of tunneling spectroscopy of $d$-wave superconductors", Phys. Rev. Lett. 74, 3451 (1995); S. Kashiwaya, Y. Tanaka, M. Koyanagi and K. Kajimura, "Theory for tunneling spectroscopy of anisotropic 
superconductors," Phys. Rev. B 53, 2667 (1996); S. Kashiwaya and Y. Tanaka, "Tunneling effects on surface bound states in unconventional superconductors," Rep. Prog. Phys. 63, 1641 (2000).

[30] E.-A. Kim, S. Vishveshwara and E. Fradkin, "Cooper pair tunneling in singlet quantum Hall/superconductor junctions," cond-mat/0405156.

[31] D.E. Sheehy, I. Adagideli, P.M. Goldbart and A. Yazdani, "Probing d-wave pairing correlations in the pseudogap regime of the cuprate superconductors via low-energy states near impurities," Phys. Rev. B 64, 224518 (2001).

[32] I. Adagideli, D.E. Sheehy and P.M. Goldbart, "Density of states in d-wave superconductors disordered by extended impurities," Phys. Rev. B 66 140512(R) (2002). [32] U.C. Coskun, T.-C. Wei, S. Vishveshwara, P.M. Goldbart and A. Bezryadin, "h/eMagnetic flux modulation of the energy gap in nanotube quantum dots", Science 304, 1132 (2004).

[33] D. Hopkins, A. Bezryadin, D. Pekker and P.M. Goldbart, "Experimental observation of magnetoresistance oscillations in a mesoscopic superconducting phase gradiometer device," to be submitted to Science (2004).

[34] D. Pekker, P.M. Goldbart, D. Hopkins and A. Bezryadin, "Theory of magnetoresistance oscillations in a mesoscopic superconducting phase gradiometer device," to be submitted to Phys. Rev. B (2004).

[35] I. Adagideli and P.M. Goldbart, "Quantal Andreev billiards: Density of states oscillations and the spectrum-geometry relationship," Phys. Rev. B 65, 201306(R) (2002); "Quantal Andreev billiards: Semiclassical approach to mesoscale oscillations in the density of states," Int. J. Mod. Phys. B 16, 1381-1458 (2002).

[36] D. Sánchez-Portal, J.D. Gale, A. García and R.M. Martin, "Two distinct metallic bands associated with monatomic Au wires on the Si(557)-Au surface," Phys. Rev. B 65, 081401 (2002).

[37] I.K. Robinson, P.A. Bennett and F.J. Himpsel, "Structure of quantum wires in Au/Si(557)," Phys. Rev. Lett. 88, 096104 (2002).

[38] D. Sanchez-Portal and R.M. Martin, "Role of the spin-orbit splitting and the dynamical fluctuations in the Si(557)-Au surface," Phys. Rev. Lett. 93, 146803 (2004).

[39] T.-C. Wei and P.M. Goldbart, "Geometric measure of entanglement and applications to bipartite and multipartite quantum," Phys. Rev. A 68, 042307 (2003).

[40] T.-C. Wei, D. Das, S. Mukhopadhyay, S. Vishveshwara and P.M. Goldbart, "Global entanglement and quantum criticality in spin chains," submitted to Phys. Rev. Lett. (2004).

[41] D.J. Van Harlingen, T.L. Robertson, B.L.T. Plourde, P.A. Reichardt, T.A. Crane, and J. Clarke, "Decoherence in Josephson-junction qubits due to critical-current fluctuations", Phys. Rev. B 70, 064517 (2004).

\section{Complex Materials, Interfaces, and Their Field Responses}

Task 1: Structure and dynamics of soft material fluids under external fieldsPrimary Participants: P. Bohn, D. Dlott, A. Gewirth, S. Granick, D. Leckband, E. Luijten, T. Martínez, A. Wieckowski, G. Wong. 
Task 2: Functionality resulting from controlled nanoscale architecture of soft materialsPrimary Participants: P. Bohn, D. Dlott, A. Gewirth, S. Granick, D. Leckband, E. Luijten, T. Martinez, P. Selvin, G. Wong.

This research cluster has led to 344 publications, including 4 in Science, 15 in J. Am. Chem. Soc., and 27 in Physical Review Letters. Among the students who participated, more than $60 \mathrm{Ph}$.D. degrees were awarded. Thematically, results from previous support revolve around understanding buried interfaces, interfacial dynamics, the design and characterization of novel surface nanostructures, and around understanding the origins of spontaneous order induced by materials dispersed in aqueous fluids.

This group has innovated several major new scientific approaches to the study of soft materials. Among the most important functions of soft materials are those that occur when they are placed in sliding contact ${ }^{[1]}$. We have applied stateof-the-art laser spectroscopy techniques to study liquids at interfaces led to notable advances regarding the solid-liquid interface with exciting implications for understanding friction and lubrication at the molecular scale $[2,3]$. This was investigated using newly-invented experimental platforms that combine the ensemble-averaged measurement of friction with direct in situ measurements of molecular diffusion and chain orientation using laser technology. Prior molecularlevel studies of friction relied on the experimental side on force measurements ${ }^{[1]}$; here, for the first time, they were augmented by direct investigation at the molecular level. Specifically, translational diffusion of fluorescent dyes at nanomolar

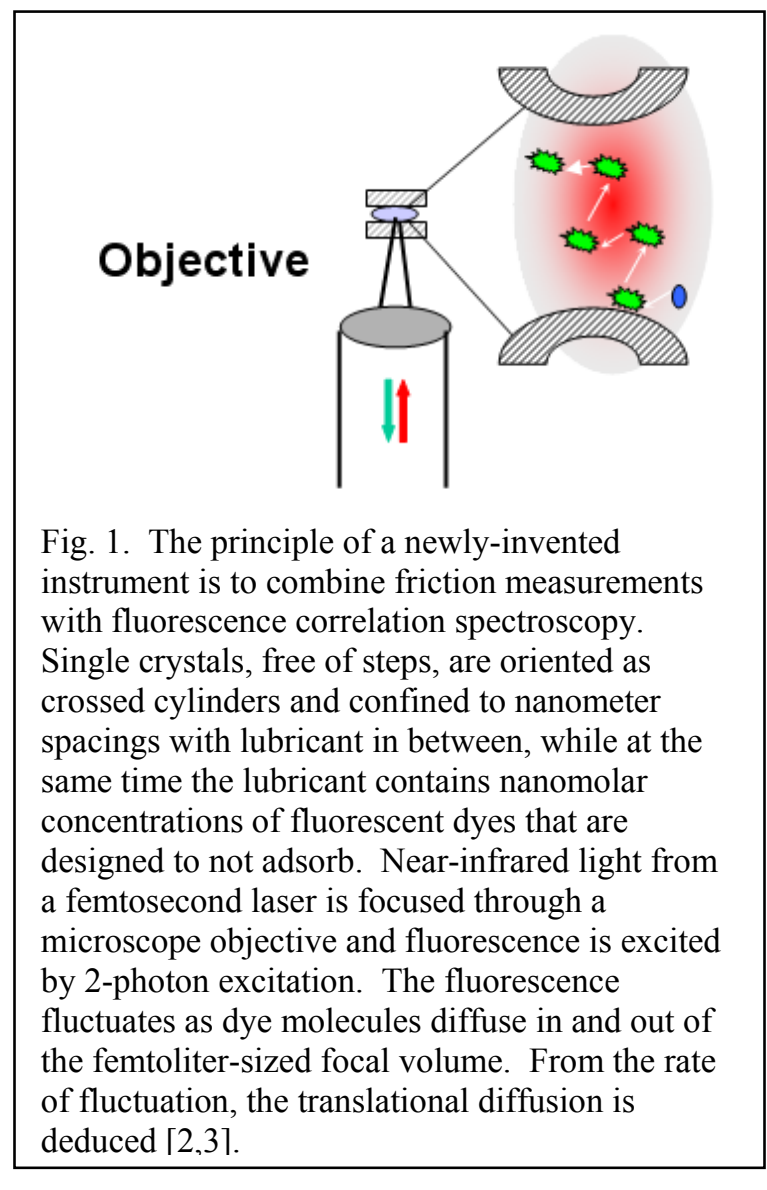
concentration was measured within films of nanometer thickness, thus enabling the contrast of viscosity and diffusion within molecularly-thin fluid films (Fig. 1). Diffusion depended remarkably strongly on the local pressure squeezing on the film such that the large deduced activation volume indicated a collective process. The significance was to identify massively heterogeneous dynamics. The conclusion emerged that friction, an ensemble average property, masks a rich pattern of heterogeneous molecular mobility. Looking to the future, the feasibility of time-correlated fluorescence measurements in a single confined contact opens up a class of experiments not previously possible regarding molecular understanding of lubrication, adhesion, transport in colloidal dispersions, and 
the liquid interfaces present in other confined environments important to the science missions of the Department of Energy.

The collaborations that underpin this work also led us to enlarge these problems to investigate how energy is dissipated not just in friction but also through temperature gradients $^{[4]}$. Studies of heat flow were performed on femtosecond time scales using aqueous dispersed organic mesophases as explicit models of solid-liquid interfaces important for polymeric materials. In a micelle environment the flow of vibrational energy across a molecular monolayer separating water from $\mathrm{CCl}_{4}$ was measured using vibrational spectroscopy. In reverse micelles consisting of $\sim 35$ water molecules surrounded by a monolayer of AOT surfactant, a femtosecond IR pulse excited water vibrations. A Raman probe pulse monitored the flow of vibrational energy within 10 picoseconds, from water to the AOT sulfate head group and AOT CO, through the AOT alkane tail and out to the $\mathrm{CCl}_{4}$. The results were quite different than would be expected for ordinary heat transfer. The main point is that the shape and detailed chemical makeup of the molecules really matter, even for heat flow across a single molecular layer. This points the way to understanding in detail the roadmap of how energy is redistributed when local spots of a complex system are driven out of equilibrium.

Materials also degrade by the process known as corrosion ${ }^{[5]}$. To understand the fundamentals of corrosion, especially how water organizes at metal surfaces, infrared visible sum-frequency generation spectroscopy (SFG) was employed to show how the structure of water at the Ag-water interface depends on the presence of electrolyte ${ }^{[6]}$. In the absence of electrolyte, the $-\mathrm{OH}$ stretching mode of water at the $\mathrm{Ag}$ surface was consistent with water that exhibits low levels of hydrogen bonding. However, in the presence of $0.1 \mathrm{M} \mathrm{KF}$, vibrational modes were observed which implies that water has both surface coordination and anion-induced hydrogen bonding above the surface. The significance is to show how electrolyte serves to organize the structure of water at a metal surface. The main point is to quantify the relationship between water structure and ions that are dissolved in it. This was the first such study to establish this proof-ofconcept. Looking to the future, it points the way as to how one can elucidate quantitatively these important questions as a function of anion and cation size, charge, structure, electrochemical potential, and extent of reaction as an electrochemical reaction develops in time. These issues lie at the heart of materials responses involving heterogeneous charge/electron transfers.

A second major area of activity during the previous grant period concerns the growth, manipulation, and use of surfacerelated organic nanostructures as a means for controlling materials growth ${ }^{[7]}$ (Fig. 2).

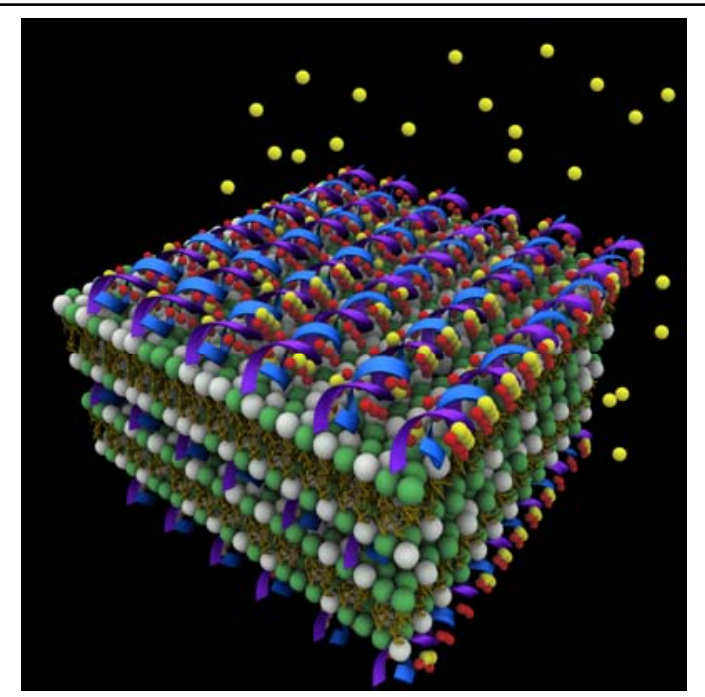

Fig. 2. Strong electrostatic interactions align the templated CdS (002) polar planes parallel to the orientation of the negatively charged sugarphosphate DNA backbone. Molecular details on the surface of the DNA molecule are imprinted onto the inorganic crystal structure [8]. 
We showed that crystallographic control of the growth of inorganic nanostructures is possible using synthetic biomolecular templates comprised of anionic DNA and cationic membranes, which self-assemble into a multi-lamellar structure where a periodic one dimensional (1D) lattice of parallel DNA chains is confined between stacked two dimensional (2D) lipid sheets. We have organized $\mathrm{Cd}^{2+}$ ions within the interhelical pores between DNA strands, and subsequently reacted them with $\mathrm{H}_{2} \mathrm{~S}$ to form $\mathrm{CdS}$ nanorods of controllable widths and crystallographic orientation. The strong electrostatic interactions align the templated CdS (002) polar planes parallel to the negatively charged sugarphosphate DNA backbone, which demonstrates a profound example of materials recognition by the DNA molecule whose motifs are then imprinted onto the inorganic crystal structure. Looking to the future, this demonstrates a strategy by which to aim to achieve full control of crystal structure using different conformations of biopolymers in the template ${ }^{[8,9]}$.

Many of the materials applications of soft materials involve chemical reactions. Extending this idea, the capability was demonstrated to control the rate of a chemical reaction spatially and to use that capacity to map reactions onto surfaces ${ }^{[10]}$. Building on spatiotemporal control of composition by controlling the energetics of electrosorption, electropolymerization reactions were used to produce laterally-varying thickness gradients of polyacrylic acid (PAA) formed by $\mathrm{Zn}$ (II)-catalyzed electropolymerization of acrylic acid in the presence of an in-plane electrochemical potential gradient applied to $\mathrm{Au}$ working electrodes. The in-plane thickness variations of the PAA gradients, both static and dynamic, can be correlated very well with predictions of the Butler-Volmer equation at small absolute overpotentials. At sufficiently large (negative) overpotentials, mass transport dominates, and the thickness reaches a plateau value independent of local potential. The capability of spatial control of surface chemical reactions forms the basis of exciting new biosensing applications.

In parallel studies, direct force measurements show the $\mathrm{pH}$-dependent interfacial properties of novel end-grafted copolymers ${ }^{[11]}$. This sets the stage for studies to define fundamental design requirements for controlling biological activity of polymer films and surface coatings.

Closely related is the
question of how surface reconstruction of soft materials competes with the rate of adsorption. In a series of studies, supported phospholipid bilayers were employed as substrates for adsorption of a weak polyelectrolyte polymer, whose time-dependent ratio of charged to uncharged functional groups served to probe the local dielectric environment of the interface (Fig. 3). Chains that
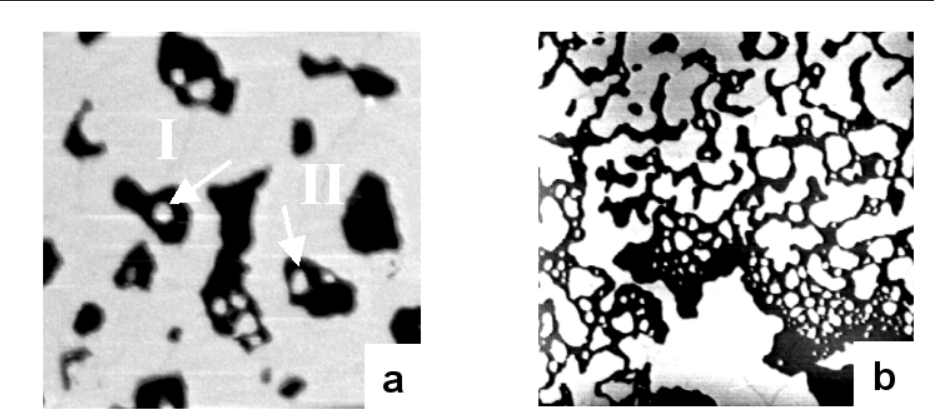

Fig. 3. AFM images of gel phase of the supported phospholipid bilayer, DMPC, (a) without $(3 \times 3 \mu \mathrm{m})$ and (b) with $(5 \times 5 \mu \mathrm{m})$ adsorbed polymethacrylic acid. Dark color is the solid substrate, light color is the lipid bilayer. Morphology of the lipid bilayer is altered by adsorption [13]. 
encountered sparsely-covered surfaces spread to maximize the number of segmentsurface contacts at rates independent of the molar mass. Surface reconstruction rather than molar mass of the adsorbing molecules appeared to determine the rate of spreading. The significance is the stark contrast with well-known views of polymer adsorption onto surfaces whose structure is "frozen" and unresponsive, and is relevant not just from biological and biophysical standpoints but also to the materials science of phospholipid bilayers $^{[12,13]}$.

Materials applications commonly involve aqueous environments. A third related theme of intense activity has revolved around understanding the structure of water and dissolved ions at a heterogeneous interface. In contradiction to conventional intuition and widely employed mean field theories ${ }^{[14]}$, experiments now show that it is possible to get attractive interactions between two objects with the same charge ${ }^{[15]}$. Actin, a model biological polyelectrolyte, was studied in order to experimentally establish the microscopic mechanism for this neglected form of like-charge attraction. The unanticipated result was obtained that the counterions organize into charge density waves on the surface of actin (a long sought confirmation of theory). Moreover, a new and unexpected coupling was discovered between these density waves and twist distortions of the polymer which enhances the attraction ${ }^{[8]}$. These ion mediated interactions are strongly attractive and appear to hold profound implications for all forms of materials assemblybiological or synthetic - in aqueous media. To understand the collective dynamics of this phenomenon constitutes an exciting agenda for future work.

A breakthrough was achieved regarding the dynamical structure of water confined between a hydrophilic surface on one side, and a hydrophobic surface on the other. We call this a Janus interface (Fig. 4) ${ }^{[16]}$. Stable films formed of nanometer thickness whose responses to shear deformations were extraordinarily noisy. The power spectrum of this noise was quantified. In addition, the frequency dependence of the complex shear modulus was found to be a power law, indicating a distribution of relaxation processes rather than a single dominant one.

Theory and computation are indispensable in understanding soft material fluids that are driven by electrostatic interactions or are governed by processes at widely varying length scales. In the field of Monte-Carlo simulation of these difficult questions, a rejection-free

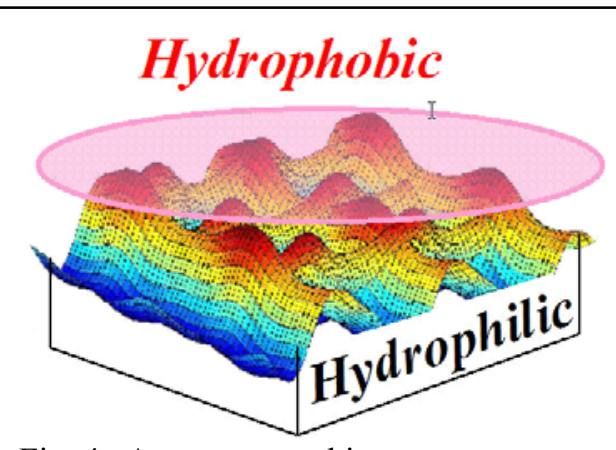

Fig. 4. A nanometer-thin gas gap appears to form spontaneously when an extended hydrophobic surface is immersed in water. Water dewets the hydrophobic side of the interface but the hydrophilic side constrains water to be present, resulting in a flickering, fluctuating complex. This has evident connections to understanding the long-standing question of the structure of aqueous films near a hydrophobic surface $\lceil 161$. geometric cluster algorithm (GCA) was invented which creates an "artificial motion" by moving a group of particles such that the newly-generated configuration is guaranteed to 
be Boltzmann-compatible (Fig. 5). This GCA yields an efficiency improvement of many orders of magnitude $\left(>10^{5}\right)$ for the simulation of complex fluids ${ }^{[17]}$.

A special apparatus that permits simultaneous electrochemical and vibrational sum-frequency generation (SFG) spectroscopy measurements was developed. The combination of high-speed data acquisition (5 spectra/s) resulting from broadband multiplex detection with an ideal thin-layer electrochemical geometry is an important advance over previous technologies and stands as a important new tool with which electrodic phenomena associated with corrosion and fuel cell catalysis can be studied ${ }^{[18]}$. Computer analysis of hundreds of SFG spectral

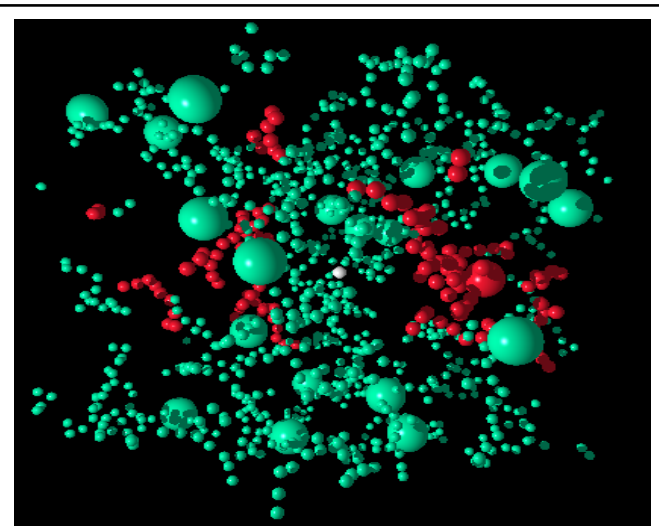

Fig. 5. A new algorithm that not only greatly accelerates the simulation of large classes of complex fluids, but also is anticipated to play a seminal role in the further advancement of Monte Carlo techniques for fluid simulations [17]. lineshapes obtained in 60 s voltage scans was used to understand microscopic details of molecule-electrode interactions and dynamics of $\mathrm{CO}$ on $\mathrm{Pt}^{[19]}$. This new instrumentation is now capable of accessing surface kinetics and surface diffusion commensurable with rates of surface poisoning in fuel cells.

Molecular simulation techniques based on direct solution of the electronic Schrödinger equation simultaneously with molecular dynamics ("ab initio MD") hold out the most promise for realistic chemical pictures of reactivity in soft material fluids and complex environments ${ }^{[20-22]}$. However, these methods are computationally very challenging, calling for the development of flexible methods which can describe bond rearrangement accurately for less computational cost. We have developed new multireference reparameterized semiempirical methods and applied them in conjunction with hybrid quantum mechanics/molecular mechanics (QM/MM) techniques to the most realistic

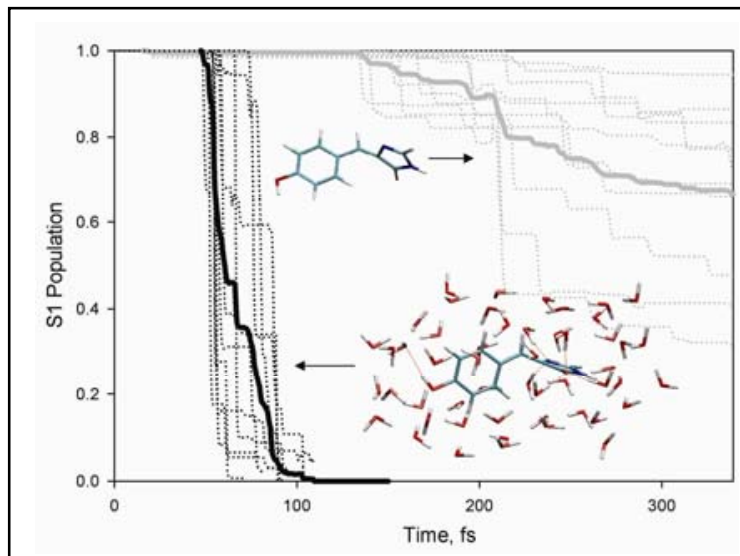

Fig. 6. Dramatic shortening of excited state lifetime in GFP chromophore by aqueous solvation. These are the first calculations to treat conical intersections, the photochemical analog of the transition state, in solvated environments with atomistic detail [24].

studies to date of electronic excited state dynamics in solution ${ }^{[23,24]}$. The effects of solvation can be dramatic, leading to an order of magnitude decrease of the electronic excited state lifetime in the chromophore of Green Fluorescent Protein (Fig. 6).

How to measure the scientific impact and significance of these results from previous support? In addition to the number and high quality of the Ph.D. students whom we have graduated, and the number and high quality of our publications in blue-ribbon scientific journals, it is worth noting that this research has also seen considerable mention in the 
popular science press. Among the science journals, pieces of this research were featured in Physics Today, Chemical and Engineering News, and APS News. Aspects of this research have also seen wide mention in the science sections in numerous other more popular newspapers and journals.

\section{Selected Publications:}

[1] B. N. J. Persson, Sliding Friction: Principles and Applications, 2nd Ed. (Springer, New York, 2000).

[2] A. Mukhopadyay, J. Zhao, S. C. Bae, and S. Granick, "Contrasting Diffusion and Viscosity in Molecularly-Thin Films," Phys. Rev. Lett. 89, 136103 (2002).

[3] A. Mukhopadyay, J. Zhao, S. C. Bae, and S. Granick, "How Confined Lubricants Diffuse During Shear," Phys. Rev. Lett. 93, 236105 (2004).

[4] J. C. Deak, Y. Pang, T. D. Sechler, Z. Wang, and D. D. Dlott, "Vibrational Energy Transfer Across a Reverse Micelle Surfactant Layer," Science 306, 473 (2004). [5] A. J. Bard and L. R. Faulkner, Electrochemical Methods: Fundamentals and Applications, 2nd Ed. (John Wiley \& Sons, Inc, New York, 2001).

[6] Z. D. Schultz and A. A. Gewirth,"Electrolyte-induced Organization of Water at the Electrified Metal-Liquid Interface" J. Am. Chem. Soc submitted (2005).

[7] See for example, S. Mann, Biomineralization: Principles and Concepts in

Bioinorganic Materials Chemistry (Oxford University Press: Oxford, 2002); C. J.

Brinker, Curr. Opin. Colloid Interface Sci. 3, 166 (1988); A. Firouzi et al., Science 267, 1138 (1995); S. I. Stupp and P. V. Braun, Science 277, 1242 (1997).

[8] H. Liang, T. Angelini, P. V. Braun, G. C. L. Wong, "Roles of anionic and cationic template components in biomineralization of CdS nanorods in self-assembled DNAmembrane complexes", J. Am. Chem. Soc. 126, 14157 (2004).

[9] L. Yang, H. Liang, T. Angelini, J. Butler, R. Coridan, J. X. Tang, G. C. L. Wong, "Selfassembly of virus membrane complexes", Nature Materials, 3, 615 (2004).

[10] X. Wang and P. W. Bohn, "Anisotropic In-Plane Gradients of Poly(Acrylic Acid) Formed by Electropolymerization with Spatiotemporal Control of the Electrochemical Potential," J. Am. Chem. Soc. 126, 6825 (2004).

[11] X. Zhu, J. DeGraaf, F. Winnik, D. Leckband, "pH-responsive PNIPAM-N-Glycine Copolymer: Direct Measurements of Interfacial Forces," Langmuir 20, 1459 (2004).

[12] F. Xie and S. Granick, "Phospholipid Bilayers as Substrates for Polymer Adsorption," Nature Materials 1, 129 (2002).

[13] A. F. Xie, R. Yamada, A. Gewirth, and S. Granick, "Materials Science of the Gel to Fluid Phase Transition in a Supported Phospholipid Bilayer," Phys. Rev. Lett. 89, 246103 (2002); Z. Feng, S. Granick, and A. A. Gewirth, "Modification of a Supported Lipid Bilayer by Polyelectrolyte Adsorption," Langmuir (2004).

[14] W. M. Gelbart, R. F. Bruinsma, P. A. Pincus and V. A. Parsegian, "DNA-Inspired Electrostatics," Physics Today,53 (2000) 38; Y. Levin, "Electrostatic Correlations: From Plasma to Biology,” Rep. Prog. Phys., 65 (2002) 1577; A. Y. Grosberg, T. T. Nguyen, and B. I. Shklovskii, "The Physics of Charge Inversion in Chemical and Biological Systems," Rev. Mod. Phys. 74, 329 (2002). 
[15] T. Angelini, H. Liang, W. Wriggers, G. C. L. Wong, "Like-charge attraction between polyelectrolytes mediated by counterion charge density waves", Proc. Nat. Acad. Sci. USA 100, 8634-8637 (2003).

[16] X. Zhang, Y. Zhu, and S. Granick, "Hydrophobicity at a Janus Interface," Science 295, 663 (2002).

[17] J. Liu and E. Luijten, "Rejection-free geometric cluster algorithm for complex fluids," Phys. Rev. Lett. 92, 035504 (2004); J. Liu and E. Luijten, "Stabilization of Colloidal Suspensions by Means of Highly Charged Nanoparticles," Phys. Rev. Lett. 93, 247802 (2004).

[18] L. J. Richter, T. P. Petralli-Mallow, and J. P. Stephenson, "Vibrationally Resolved Sum-Frequency Generation with Broad-Bandwidth Infrared Pulses "Opt. Lett. 23, 1594 (1998); S. Baldelli, N. M. Markovic, P. N. Ross, Y. R. Shen, and G. A. Somorjai, "Sum Frequency Generation of CO on (111) and Polycrystalline Platinum Electrode Surfaces: Evidence for SFG Invisible Surface CO "J. Phys. Chem. B 103, 8920 (1999).

[19] G. Q. Lu, A. Lagutchev, D. D. Dlott, and A. Wieckowski, "Quantitative vibrational sumfrequency generation spectroscopy of thin layer electrochemistry: $\mathrm{CO}$ on a $\mathrm{Pt}$ electrode," Surf. Sci. submitted 2005).

[20] R. Car and M. Parrinello, "Unified Approach for Molecular Dynamics and Density Functional Theory," Phys. Rev. Lett. 55, 2471 (1985).

[21] H. B. Schlegel, J. M. Millam, S. S. Iyengar, G. A. Voth, A. D. Daniels, G. E. Scuseria, and M. J. Frisch, "Ab Initio Molecular Dynamics: Propagating the Density Matrix with Gaussian Orbitals," J. Chem. Phys. 114, 9758 (2001).

[22] M. Ben-Nun, J. Quenneville, and T. J. Martinez, "Ab Initio Multiple Spawning: Photochemistry from First Principles Quantum Molecular Dynamics," J. Phys. Chem. A 104, 51615 (2000).

[23] A. Toniolo, A. L. Thompson and T. J. Martinez, "Excited State Direct Dynamics of Benzene with Reparameterized Multireference Semiempirical Configuration Interaction Methods," Chem. Phys. 304, 133 (2004).

[24] S. Olsen, A. Toniolo, C. Ko, L. Manohar, K. Lamothe and T. J. Martinez, "Computation of Reaction Mechanisms and Dynamics in Photobiology," in

Computational Photochemistry, Ed. M. Olivucci (Amsterdam, Elsevier, in press). 